Research Square
Preprints are preliminary reports that have not undergone peer review.

They should not be considered conclusive, used to inform clinical practice, or referenced by the media as validated information.

\title{
Exploring the Modulatory Mechanism of Eleng Capsule in the Treatment of Endometriosis Based on Integrated Strategy of Transcriptomics Combined With Network Pharmacology
}

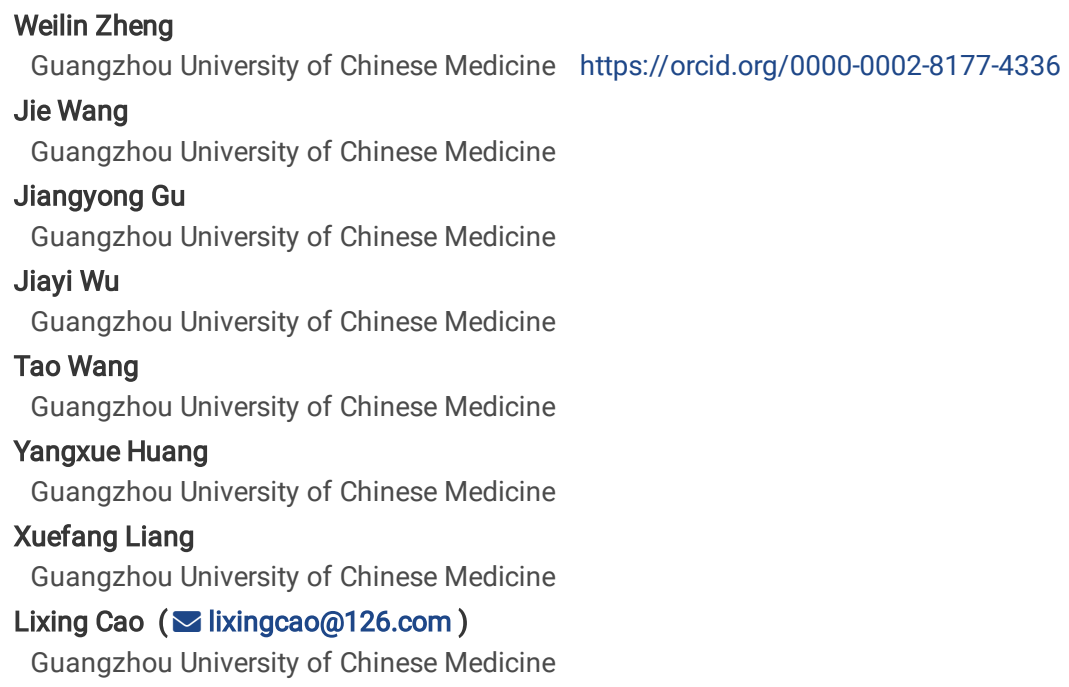




\section{Abstract}

Background

Endometriosis causes severe chronic pelvic pain and infertility. Chinese medicine plays an active role in the treatment of endometriosis. ELeng Capsule(ELC) is a Chinese medicine formula used for the treatment of endometriosis for several years. The previous studies have shown that ELC inhibits endometriosis. However, the mechanisms of action of ELC have not been characterized. In this study, network pharmacology and mRNA transcriptome analysis were used to study various therapeutic targets in ELC.

Methods

Ultra-performance liquid chromatography/quadrupole time-of-flight mass spectrometry analysis(UPLC-Q-TOF/MS) was used to identify the compounds in ELC.And network pharmacology was used to analyze the network of targets and identified compounds of ELC. Apoptosis was assessed using the terminal deoxynucleotidyl transferase (TdT) deoxyuridine 5'-triphosphate (dUTP) nick-end labeling (TUNEL) assay.The protein expression of VEGFA,VEGFB,VEGFC and $a-S M A$ in the ectopic endometrium were identified by immunohistochemistry(IHC). The level of VEGFA,VEGFB and IL1- $\beta$ in serum were used by ELISA. Further, RNA-sequencing was used to identify differentially expressed genes (DEGs) in ELC. Biological functions and pathways were determined through the Gene Ontology (GO) and Kyoto encyclopedia of genes and genomes pathway(KEGG) analyses. In addition, Gene Set Enrichment Analysis (GSEA) analysis was used to further analyze the genetic network and modular genetics.

Results

We had identified 26 new bioactive compounds in ELeng Capsule by UPLC-Q-TOF/MS analysis. The KEGG pathways of ELC associtaed targets related to neuroactive ligand-receptor interaction, the toll-like receptor signaling pathway, and the vascular endothelial growth factor signaling pathway by network pharmacology analysis.Further, ELC could induce cell apoptosis, inhibit angiogenesis through reduce the expression of VEGFA and VEGFC, and inhibit the fibrosis through reduce the expression of a-SMA in ectopic lesions $(P \otimes 0.05)$. In addition, the development of endometriosis in the rat model may be related to mechanisms of inflammation,epithelial-mesenchymal transition(EMT) by RNA-sequencing. And the targets in the treatment of ELC were predominantly associated with actin and cytoskeleton,EMT,focal adhesion,and inflammatory immunity based on the DEGs analysis. In additon, the GSEA analysis showed that the treatment of ELC was associated with the Notch signaling pathway, Hippo signaling pathway, etc.

Conclusion

ELeng Capsules exerted beneficial effects against endometriosis, potentially by induce apoptosis,modulating the angiogenesis,cytoskeleton, epithelialmesenchymal transition(EMT), and cell junction-associated pathways,etc. These findings could provide evidence for an innovative treatment strategy and novel therapeutic targets for endometriosis.

\section{Background}

Endometriosis causes severe chronic pelvic pain and infertility. Currently, the treatment of endometriosis is mainly based on surgery and pharmacological treatment, and long-term treatment management programs are continuously optimized[1]. Endometriosis is a disease under the influence of multiple factors. The mechanism of endometriosis is complex and associated with inflammation, immunity, angiogenesis, epithelial-mesenchymal transition (EMT), etc[2][3]. TCM plays an active role in the treatment of endometriosis such as endometriosis, chronic pelvic pain, abnormal uterine bleeding, dysmenorrhea, and infertility, which is associated with pain and infertility, by regulating inflammation, immunity, and angiogenesis[1, 4].

The Chinese preparation ELeng Capsule (ELC), as a hospital preparation, has been used in the Guangdong Provincial Hospital of Chinese Medicine (Guangzhou, China) to relieve the symptoms of endometriosis-associated pain and dysmenorrhea for nearly 20 years. The previous clinical experience, modern clinical practice and animal experiments of ELC have shown that ELC could reduce endometriosis-associated pain and inhibit the development of endometriosis through inhibits adhesion and regulate inflammation and immunity $[5,6]$. However, the mechanisms of action of ELC have not been characterized. It is significance to discover the multi-target regulatory mechanism of ELeng capsule with new methods.

Traditional Chinese medicine (TCM) compounds exist in complex mixtures and may contain thousands of compounds. Therefore, it is difficult to explain the principle of compatibility of Chinese medicine ingredients and analyze relevant results. Network pharmacology can predict the profiles of targets and pharmacological actions of herbal compounds to reveal "drug-genes/targets-disease". This approach could be used to determine the herbal ingredients and their related properties, as well as compound-target and target-disease relationships,which will improve current drug discovery strategies[7-9]. At the same time, the development of multi-omics also provides new tools for research on TCM. The expression of mRNA examined through microarray or high-throughput RNA-sequencing (RNA-seq) has been used to reveal molecular mechanisms and explore biomarkers for diagnosis and treatment[10, 11]. Transcriptome-based studies of endometriosis could provide the basis for clarifying the therapeutic mechanisms of drugs[12]

In the study, we first predicted the potential targets of ELC involved in endometriosis. Then, endometriosis model rats were used to validate some of the potential targets in ELeng capsules. Further,the RNA-sequencing were performed to identify genes whose expression is regulated by ELC treatment in endometriosis model, and find the potential multi-targets regulation of ELC.We aim to provide a reliable way for subsequent experimental verification and new drug research and development.

\section{Methods}


It is summarized as follows: (A)The compounds of ELeng capsules were identified by UPLC-Q-TOF/MS. (B)Network pharmacology were used to analysis the the compounds-targets and endometriosis associated targets network of ELeng Capsules and validation of endometriosis model rats. (C)A model of endometriosis was established through allotransplantation in rats and used to verify the core targets. (D)RNA-sequencing was used to identify differentially expressed genes (DEGs). Up-regulated and down-regulated genes of the endometriosis model ectopic endometrium versus control eutopic endometrium, endometriosis model ectopic endometrium versus ELC-induced endometriosis model ectopic endometrium were identified. Biological functions and pathways were determined through the Gene Ontology and Kyoto encyclopedia of genes and genomes pathway analyses. Gene Set Enrichment Analysis (GSEA) analysis was used to further analyze the genetic network and modular genetics.

\section{The Preparation of ELeng Capsule}

ELC, as one kind of hospital preparation, are provided by the pharmaceutical department of Second Affiliated Hospital of Guangzhou University of Chinese Medicine(Guangdong Province Hospital of Chinese Medicine) Pharmaceutic (Guangzhou, China). ELC was formed by E'zhu(Curcuma phaeocaulis),Sanleng

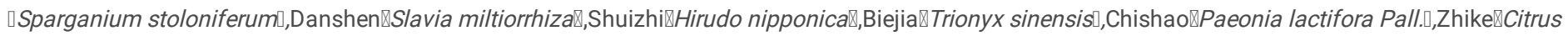
aurantium \and Danggui囚Angelica laxifoliata囚.The Original medicinal materials purchased from Guangdong KangMei Pharmaceutical Co.,Ltd. Guangdong KangMei Pharmaceutical Co., Ltd. undertook the formal identification of the plant material used in the study. The quality of the raw herbs was controlled according to the Pharmacopoeia of the People's Republic of China (2010). The aqueous extract of ELC was prepared through the alcohol extraction-water precipitation method. The patent certificate number is No.432493. The ELC was obtained with the water extraction and alcohol precipitation method.Each content of capsule is $0.45 \mathrm{~g}$. The herbs in ELeng Capsules were showed in Table 1.

\section{UPLC-Q-TOF/MS assay of the concentration of ELeng Capsules}

For the preparation of compounds, $20 \mathrm{ml}$ of $50 \%$ ethanol solution was added to $1 \mathrm{~g}$ of medicinal powder. The mixture was ultrasonicated for 30 min, centrifuged at $1.2 \times 10^{4} \mathrm{rpm}$ for $5 \mathrm{~min}$, and the supernatant was removed. The chromatographic conditions were as follows: the UPLC device was an Agilent 1290 UPLC and the column was an Agilent SB-C $18,2.1 \times 100 \mathrm{~mm}, 1.8 \mu \mathrm{m}$. The column temperature was $30^{\circ} \mathrm{C}$ and the injection volume was $5 \mu \mathrm{l}$. The detection wavelength was $254 \mathrm{~nm}$.Flow ratio and flow rate: phase $A$ is $0.1 \%$ formic acid aqueous solution,and phase $B$ is acetonitrile. Gas chromatography-mass spectrometry (GC-MS) was performed using an Agilent 7890A/5975C. The column was an Agilent HP-5MS $30 \mathrm{~m} \times 250 \mu \mathrm{m} \times 0.25 \mu \mathrm{m}$. The inlet temperature was $250^{\circ} \mathrm{C}$, ion source temperature was $230^{\circ} \mathrm{C}$, and quadrupole temperature was $150^{\circ} \mathrm{C}$. The compounds were tentatively characterized based on their retention time, mass accuracy of precursor ions, MS/MS spectra and fragmentation pathways, referring to the SCIEX natural products HR-MS/MS Spectral Library and previous literatures.The conditions of UPLC-Q-TOF/MS are listed in Table 2.

\section{Network pharmacology analysis of ELeng Capsule}

\section{Construction of the compound-target in ELC and disease-target databases}

Compounds of ELC were compiled from from the STITCH database, the traditional Chinese medicine systems pharmacology (TCMSP) database(http://tcmspw.com/tcmsp.php),universal natural product database (UNPD)[13]. The structures of natural compounds in the medicinal plants were retrieved from the Pubchem dataset (https://www.ncbi.nIm.nih.gov/pccompound/).The druggability of each candidate was analyzed according to its oral bioavailability (OB) and drug-likeness (DL) indices. To better understand the processes of ELC, we performed GO enrichment and KEGG pathway enrichment analyses of ELC associated targets. We used the web-based search engine, DAVID, to determine over-represented GO terms and KEGG pathways with thresholds of an enrichment score $>2$, count $>5$ and $P<0.05$.

\section{The targets of endometriosis}

We also collected the targets of endometriosis. Mechanisms and targets related to endometriosis were selected from the Genecard(https://www.genecards.org/) and Genbank(https://ncbiinsights.ncbi.nlm.nih.gov/tag/genbank/) database.The targets and proteins were researched in the UniProt (https://www.uniprot.org). The three-dimensional structures of proteins related to endometriosis were obtained from the Research Collaboratory for Structural Bioinformatics Protein Data Bank (PDB)(www.rcsb.org/pdb/home/home.do).

\section{Molecular docking}

Molecular docking, combined with the active components identified in ELC, to construct the network of small molecule compounds and targets for the prediction of compounds and targets regulation at the protein level. All molecular three-dimensional structures of active ingredients were obtained from the PubChem Compound Database (http://www.ncbi.nlm.nih.gov/pccompound) in mol2 format. The AutoDock 4.2.6 program was used to perform virtual screening and the binding free energy-based docking score $(p K i)$ was used to evaluate the affinity between each compound and protein. 180 endometriosisrelated targets were selected for auto docking. We screened the "compound-target" with a molecular docking result of binding energy <-10 kcal/mol. Further,we established a disease-target-compounds network based on currently available small molecule compounds and endometriosis-related targets for endometriosis. 
The online search tool for recurring instances of neighboring genes (STRING, Version 9.1) (http://www.stringdb.org/) to predict the protein-protein interactions. The compounds-targets networks were constructed using Cytoscape software 3.7.2(http://cytoscape.org/). Related parameters were calculated to detect significant nodes[14]

\section{Animal model and treatments}

This study used female Sprague-Dawley (SD) rats (age: $8 \pm 1$ weeks, weight: $220-230 \mathrm{~g}$ ). The rats are from the Experimental Animal Center of Guangdong Province(Guangzhou,Guangdong,China), and certificate number is 44007200054328 . The rats were housed at $20 \pm 2^{\circ} \mathrm{C}$ on a $12-\mathrm{h}$ light/dark cycle, with ad libitum access to food and water,raised in the Laboratory Animal Center of the The Second Clinical College of Guangzhou University of Chinese Medicine(Guangzhou,Guangdong,China). The rats were housed five per cage. Animals, and the protocols protocols were approved by the Guangdong Provincial Hospital of Chinese Medicine Committee on the Use of Live Animals for Teaching and Research (SZY2016007). And disposal methods were in accordance with animal ethics standards.

\section{Surgical operation}

A model of endometriosis was established through allotransplantation in rats. All operational procedures were conducted under sterile conditions. The rats were anesthetized with $3 \%$ pentobarbital sodium prior to performing a vertical incision in the abdomen for tumor transplantation. The right uterus of each rat was removed and immediately placed in a saline solution. The endometria were separated from the myometria and cut into $0.5 \times 0.5 \mathrm{~cm}$ pieces. The endometria were sutured onto the peritoneum close to blood vessels in each abdominal wall using a 5-0 absorbable suture. The incision was closed and disinfected, and the animals were allowed to recover from anesthesia. After transplantation (28 days), the growth of the ectopic endometrium was observed via gross and microscopic examination. Endometriosis rats model established are following criteria that viable and well-vascularized endometrial explants[15].The volume of ectopic endometrium were detected by Vernier caliper with volume formula (length $\times$ width $\times$ height $\times 0.52$ ).

The 40 endometriosis SD rats were randomly divided into four groups: the ELeng Capsule low-dose group(group A, 0.5g/kg/d of ELeng Capsule), the ELeng Capsule high-dose group ( $2 \mathrm{~g} / \mathrm{kg} / \mathrm{d}$ of ELeng Capsule), the middle group ( $1 \mathrm{~g} / \mathrm{kg} / \mathrm{d}$ of ELeng Capsule), the low group ( $0.5 \mathrm{~g} / \mathrm{kg} / \mathrm{d}$ of ELeng Capsule), and the model group (group E, $10 \mathrm{ml} / \mathrm{kg} / \mathrm{d}$ of $0.9 \% \mathrm{Nacl}$ ). The mixed suspension was orally administered once daily.In addition, ten SD rats were selected as the control group and fed routinely.Based on the previous research basis, we choose the equivalent and human dose to RNA-sequence. At the end of ELC administration, eutopic endometria from the control group and ectopic endometria from the other groups were collected and fixed in paraformaldehyde,and parts of the tissues were maintained at $-80^{\circ} \mathrm{C}$ for future research. The volumes of ectopic endometrial lesions in each group pre-(V1) and post-(V2) treatment were measured. The tissues were used for histopathology analyse,immunohistochemistry, RNA-sequence and quantitative-polymerase chain reaction (qPCR) validation.

\section{Euthanasia}

Intraperitoneal injection of three times the anesthetic dose of $1 \%$ sodium pentobarbital solution.

\section{Hematoxylin and Eosin(H\&E) and Masson's trichrome staining}

Sections from different groups were stained with hematoxylin and eosin. Masson's trichrome staining was used for the detection of collagen fibers in tissues. The stained areas of the sections were observed under an optical microscope(Nikon囚Japan) and NIS-Elements.Fibrosis analysis was performed using Image $\mathrm{J}$ software to analyze the proportion of blue staining (fibrosis staining).

\section{Electron Microscopic Analysis}

These tissue samples were fixed immediately with $1 \%$ glutaraldehyde and $4 \%$ formalin for 6 hours at $4^{\circ} \mathrm{C}$ and rinsed in $0.1 \mathrm{M}$ cacodylate buffer overnight. Ultrathin sections were prepared with an Ultratome Nova, doublestained with uranyl acetate and lead citrate, and examined under an electron microscope.

\section{Terminal Deoxynucleotidyl Transferase-mediated DigoxigenindUTPNick-End Labeling Assay (TUNEL)}

The paraffin-embedded tissue sections were dewaxed in xylene and rehydrated after remaining in a $60^{\circ} \mathrm{C}$ warm case for $1 \mathrm{~h}$. The sections were washed three times, for 5 minutes per wash, with TBS. Apoptosis was detected using terminal deoxynucleotidyl transferase biotin-dUTP nick-end labeling (TUNEL) apoptosis detection kit (C1086,Beyotime Biotechnology,China) according to the manufacturer's instructions. The labeled apoptotic cells expressed green fluorescence under fluorescence microscopy. The Image $\mathrm{J}$ software was used for assessing the ratio.

\section{Monoclonal antibody and microvessel density (MVD)}

In this study, vascular endothelial cells were labeled with a CD34 monoclonal antibody and microvessel density (MVD) was counted(n=6 each group). The dilution ratio of anti-CD34(1:500, ab185732,ABcam, USA) were used. Three microvessel dense areas were selected for each slice, and the microvessels were counted by double-blind method under high power (200x).

\section{Immunohistochemical Staining}

The sections were stained by IHC to detect the expression of factors in the VEGF family and a-SMA. After the antigen was repaired, primary antibodies were added at $4^{\circ} \mathrm{C}$ for overnight, and then secondary antibodies were added at room temperature for 1 hours, avoiding light. Diaminobenzidine (DAB) was used for 
staining, and neutral gum was used to seal pieces. The antibodies were anti-VEGFA (1:000, ab81289, Abcam, USA), anti-VEGFB (1:000, ab81289, Abcam, USA) and anti-VEGFC (1:000, ab81289, Abcam, USA) ,a-SMA(1:1000, 14395-1-AP, Proteinech, USA)was used to evaluate. The Image $\mathrm{J}$ software was used for assessing the mean optical density.

\section{ELISA assay}

To expression of VEGF family and IL1- $\beta$ in blood serum, we used an ELISA kit. The samples and standard samples were diluted with distilled water and applied to ELISA plates. The VEGFA,VEGFB and IL1-ßconcentrations were determined according to the manufacturer's instructions. Absorbance levels were measured at $450 \mathrm{~nm}$ using an ELISA reader.

\section{RNA-sequencing of ELC}

\section{The design of RNA-sequencing analysis}

Dose of $1 \mathrm{mg} / \mathrm{kg}$ ELC was choose for further biological experiment because of its optimal effect in our previous study. We randomly selected transcriptomes from endometrial tissues and ectopic endometria for analyses between the model and ELC groups ( $n=4$, each group eutopic endometrium (Con_Euto), (Model_Euto) and (ELC_Euto); n=4 model group ectopic endometrium (Model_Ecto); n=5, ELC group ectopic endometrium (ELC_Ecto)). A crossover comparison was performed in the following three paired groups to identify genes that were differentially regulated in the endometriosis rat model and ELC treatment rat model: Control_Eu versus Model_Ecto groups; Control_Eu versus ELC_Ecto groups; and Model_Ecto versus ELC_Ecto groups.

\section{Gene Ontology (GO) terms and Kyoto Encyclopedia of Genes and Genomes (KEGG) analyses}

The transcriptome sequencing and analysis were conducted by OE Biotech Co., Ltd. (Shanghai, China). Raw data (raw reads) were processed using Trimmomatic[16]. Multiple hypothesis test correction for the treatment effect was performed using the false discovery rate (FDR) method. GO enrichment and KEGG pathway enrichment analyses of differentially expressed genes (DEGs) were respectively performed using R studio based on the hypergeometric distribution. Fisher's exact and chi-squared tests were applied to classify significant GO categories, and the FDR was used to correct $p$-values. The GO analysis provides three structured networks of defined terms to describe gene product attributes: cellular compartment (CC), biological process (BP), and molecular function (MF). Pathway analysis was applied to determine the significant pathways of DEGs according to the Kyoto Encyclopedia of Genes and Genomes(KEGG), MapSplice, and Reactome Functional Interaction Network and external interaction databases(Reactome databases). Fisher's exact test was used to identify significantly enriched pathways, and the threshold of significance was defined as $P<0.05$ and FDR<0.05.

\section{Gene Set Enrichment Analysis (GSEA)}

The publicly available GSEA software package (www.broad.mit.edu) was used for leading edge analysis to examine genome-wide expression profiles[17]. In this study, 1000 genes of permutations were set to generate a null distribution for the enrichment score in the hallmark gene sets and functional annotation gene sets. Nominal $P<0.05$, FDR $<0.25$, and gene set size $>100$ were defined as the cut-off criteria. The aim of this analysis was to determine whether the members of the identified gene ontology and KEGG pathways were randomly distributed throughout the ranked gene list or concentrated at the top or bottom.

\section{Trend modular analysis(STEM analysis)}

To identify genes that were differentially regulated under the conditions of endometriosis model and ELC_Ecto groups, a crossover comparison was performed in the following 3-paired groups:control group, model endometriosis rats groups and ELC treatment groups,as the Venn diagram to analysis demonstrates that they are coexpressed, respectively, in each pair of groups. Short Time-series Expression Miner (STEM) the first software program specifically designed for the analysis of short time series microarray gene expression data[18],which could also be used to analyze trends in genes in different groups.STEM software were used to analyze the trend of ectopic endometrium in control rats-model_Ecto endometrirum-ELC_Ecto endometrium group.

\section{Construction of the protein-protein interaction (PPI) network}

The Search Tool for the Retrieval of Interacting Genes/Proteins (STRING) database provides comprehensive information regarding interaction between proteins, including both predicted and experimental interactions. Subsequently, the PPI network was visualized using Cytoscape (Version 3.7.2; National Institute of General Medical Sciences, Bethesda, MD, USA[14]. The PPI network was used to filter modules based on the Molecular Complex Detection plug-in (MCODE) in Cytoscape with the following conditions: degree cut-off=2; $k$-core=2; node score cut-off=0.2; and max depth=100. The interaction of each gene with other genes was determined using the GeneMANIA software (www genemania.org)[19].

\section{Connectivity map(CMAP) analysis}

CMAP is a systematic approach developed for the discovery of functional connections among diseases, genes, and drugs through the analysis of genomewide transcriptional expression data.We used these probesets to query the CMAP 02 database (https://portals.broadinstitute.org/cmap/, updated September $12,2017)[20]$. The CMAP database was searched to identify small molecule compounds in TCM. The small molecule drugs were filtered according to the $p$ values $(P<0.05)$.

\section{Quantitative reverse transcription-PCR (qPCR)}


qRT-PCR was performed to validate the gene expression data obtained from deep sequencing. Total mRNA was extracted using the TRIzol reagent (Invitrogen, Carlsbad, CA) according to the instructions provided by the manufacturer. The first strand of cDNA was synthesized using primers designed in our laboratory. The RT product was amplified using SYBR Green on a 7500 Real-Time PCR System (Thermo Fisher Scientific Inc. Waltham, MA, USA). All samples were run in triplicate, and relative gene expression was analyzed according to the $2^{-\Delta \Delta C t}$ method. The sequencing accessions of the primers were myogenin (Myog) $\triangle S E T$ and MYND domain containing 1(Smyd1),SIX Homeobox 1(Six1),calcium voltage-gated channel subunit alpha1 S (Cacna1s), eukaryotic translation elongation factor 1 alpha 2 (Eef1a2), ryanodine receptor 1 (Ryr1), actinin Alpha 2(Actn2), myogenic differentiation 1 (Myod1), mitogen-activated protein kinase 12 (Mapk12), myosin heavy chain 4 (Myh4), .Gene expression levels were normalized to that of ACTB. The primer sequencings are shown in Table 3.

\section{Statistical analysis}

Data were analyzed using the Prism software (version 7.0;GraphPad prism, California San Diego,USA). All experimental data are presented as the mean \pm standard error of the mean. The qPCR data were analyzed using two-tailed Student's $t$-test. The F-test was used to determine whether two groups possessed equal variances. Unless otherwise indicated, $P<0.05$ denoted statistical significance.

\section{Results}

\section{UPLC-Q-TOF/MSresults for ELC}

In our previous study, GC-MS analysis was used to reveal the volatile oil components of ELC. A total of 14 compounds were identified, namely eucalyptol, Dcamphor and isoborneol, I(-)-Borneol, $a$-terpineol, $\beta$-elemene, $\gamma$-elemene, a-humulene, germacra, curcumenol, $\beta$-cyclocostunolide, curcumenone, pulmonary Zedeone, and ent-kaurene. In this study, the compounds of ELC were identified by UPLC-Q-TOF/MS. According to the multi-stage MS information combined with the data obtained from the literature and databases, 26 compounds were identified in the ELC compound preparation.Representative fingerprint chromatograms of ELC are displayed in Figure 2. The identified compouds of ELC were showed in Table 4. Combined with our previous study results, we have identified a total of 40 compounds in ELeng Capsule.These results provide identified compounds in ELC for network pharmacology analysis.

\section{Network pharmacology analysis}

\section{The compounds associated targets of ELC}

Based on the UPLC-Q-TOF/MS data, we performed a network pharmacology analysis on the related compounds of ELC and predicted the targets of compounds. Based on the data obtained from the network pharmacology-related databases, we identified 27 core compounds with 194 targets based on STITCH $\triangle T C M S P$ and UNPD datasets. The result showed that the major targets were involved in angiogenesis, inflammation, immunity, and other modules.Supplementary Material 1 showed the major compouds and targets of ELC.In addition,we had collected 1289 endometriosis-related targets from Genecard and Genbank. A total of 75 targets of ELC capsules coincided with those of genes associated with endometriosis. Following cytoHubba analysis, VEGFA,IL6,TP53,PTGS2,AKT1,MMP9,MAPK1,JUN,etc may be core targets of ELC.

\section{The analysis of GO terms and KEGG pathways}

We analyzed the GO terms and KEGG pathways affected by targets associated with ELC. The results suggest that the BP in GO terms are related to cell death, apoptosis, and proliferation. The results of KEGG pathways were mainly enriched in cancer pathway, inflammatory response, drug reaction, etc., which were consistent with the present pathologic mechanism of endometriosis. The main pathways include neuroactive ligand-receptor interaction, TLR signaling pathway, metabolism of xenobiotics by cytochrome P450, vascular endothelial growth factor (VEGF) signaling, calcium signaling, B cell receptor signaling, among others. GO and KEGG enrichment analysis suggest that the core components of ELC may be involved in the regulation of inflammation, immunity and angiogenesis modules. The major GO terms and KEGG pathways are shown in Figure 3A and 3B. The network of major targets and compounds from database is showed in Figure 3C.

\section{Molecular docking}

We further screened 180 endometriosis-related core genes and the core compounds of ELC for molecular docking. The results implied that tanshinone IIA, cryptotanshinone, rosmarinic acid, gallic acid, linoleic acid, y-elemene, palmitate, hesperetin, danshensu, palmitic acid, naringin, and tanshinone I may be compounds that play a major role in endometriosis. The hub targets related to the compounds are as follows: eukaryotic translation initiation factor 2 alpha kinase 3 (EIF2AK3), actin-beta (ACTB), prostaglandin-endoperoxide synthase 2 (PTGS2), progesterone receptor (PGR), matrix metalloproteinase 2 (MMP2), chemokine (C-C motif) ligand 3 (CCL3), signal transducer and activator of transcription (STAT1), nitric oxide synthase 2 (NOS2), intercellular adhesion molecule 1 (ICAM1), MMP9, MYC, transforming growth factor- $\beta 1$ (TGF $\beta 1$ ), and VEGFA based on molecular docking. The results of molecular docking were showed in Figure 3D and supplementary material 2.

\section{Potential mechanism of ELC in Endometriosis rat model}

\section{Pathology of ectopic lesions}

We successfully established a rat model of endometriosis. Figure 4A shows the changes in lesions after modeling. After the treatment,the average value of tissue in ELC group was lower than the model group, while the difference was not statistically significant ( $P \otimes 0.05)$ (supplementary material 3). Compared with the model group, the lesion volumes of before and after ELC treatment in ELC middle group was change significantly $(P=0.028 \otimes 0.05)$. These result showed that 
ELC may reduce the volume of ectopic lesions to a certain extent in endometriosis rats mode. The HE revealed the formation of local glands in the lesions of the model and ELC treatment rats(Figure 4B).

\section{ELeng Capsules Promoted Apoptosis in Ectopic Endometrial Tissues}

Figure $4 \mathrm{C}$ was showed the ELC on apoptosis of endometriotic tissues, as determined by TUNEL assay ( $\times 100, n=4$ each). The distribution of green fluorescence included glandular epithelium and mesenchymal cells. The nuclei of positive staining apoptotic cells emitted green fluorescent signals in the ectopic endometrium tissues. Compared with the model group, the number of apoptotic cells in ectopic lesions in high, medium and low dose of ELC were significantly increased. Compared with the model group, the apoptotic area in the middle dose group of ELC increased significantly $(P<0.05)$.And there was no statistically significant difference in the dipsion area between the high-dose group and low-dose group compared with the model group $(P>0.05)$. This result suggested that ELC could participate in the process of cell apoptosis.

Ultrastructural Features of Ectopic Endometrium in a Rat Model

The glandular epithelial cells in the model group were relatively neat and had microvilli.In the ectopic tissue cells in ELC group,the microvilli were reduced, and the mitochondria were swollen in the cytoplasm, and autophagy and apoptotic bodies were observed(Figure 4D). Figure 4D(c,d) showed the apoptotic body, and figure $4 \mathrm{D}(\mathrm{e}, \mathrm{f})$ showed the autophagosome.This result suggested that ELC treatment may be related to the regulation of autophagy and apoptosis.

\section{The effect of Eleng capsule on angiogenesis}

Figure 5A showed that CD34 was positively expressed in both the model group and the ELeng capsule group. Among them, the expression of MVD in the ectopic lesion was significantly higher than that in the eutopic endometrium, and angiogenesis was observed(n=6, $P=0.015 \otimes 0.05)$. The expression of $\mathrm{CD} 34$ in high, medium and low dose groups of ELeng capsule was significantly lower than the model group expression (Pख0.05).

Figure 5B showed the expression of VEGFA, VEGFB and VEGFC in the cytoplasm and membrane of glandular epithelial cells in ectopic lesion in endometriosis rat model.The expression of mesenchymal cells was weaker than that of glandular epithelial cells. The expression of VEGFA in middle dose and the low dose group of ELC were decreased compared with the model group $(P<0.05)$. There was no significant difference between the model group and the ELC high, middle and low doze groups of the VEGFB expression ( $P \otimes 0.05)$.Compared with the model group, the expression of VEGFC in ectopic lesions were significantly decreased in the high, middle and low dose of ELC, and the differences were statistically significant $(P<0.05)$. These results suggested that ELC could inhibit angiogenesis through inhibiting VEGFA and VEGFB expression.

\section{The Effect of Eleng Capsule in Serum VEGFA,VEGFB and IL1- $\beta$}

Compared with the control group $(34.838 \pm 1.403 \mathrm{pg} / \mathrm{ml}, \mathrm{n}=8)$, the VEGFA level in serum increased in the model group $(38.866 \pm 2.706 \mathrm{pg} / \mathrm{ml}, \mathrm{n}=8)$, and the difference was statistically significant $(P=0.008<0.05)$. Compared with the model group, the serum VEGFA level in the high-dose group $(35.345 \pm 4.205 \mathrm{pg} / \mathrm{ml}$, $\mathrm{n}=8)$ and low-dose group $(35.024 \pm 2.662 \mathrm{pg} / \mathrm{ml}, \mathrm{n}=8)$ of ELC was significantly deseased( $(* \star P=0.020, * \star \star P=0.012)$. There was no significant difference in serum of VEGFB and IL $1-\beta$ expression in different groups endometriosis model rats $₫ P \otimes 0.05 \rrbracket$. The expression level of VEGFA, VEGFB and IL-1 $\beta$ are low in serum of normal $\mathrm{SD}$ rats and endometriosis model rats. Thus the regulation effect of ELC may be mainly localized in ectopic lesions(Figure 5C and Supplementary Material 4).

\section{ELeng Capsules could reduce the local fibrosis in Ectopic lesions}

Masson's trichrome staining showed that the ectopic lesions were fibrotic. Compared with the model group, the positive area of fibrosis were decreased in the high, middle and low dose groups of ELC, and the difference was statistically significant $(P<0.05)$. The result showed that ELC could reduce the degree of fibrosis in endometriosis model rats in ectopic lesions. The results of Masson's trichrome were shown in Figure 6.

The expression of The expression of a-SMA in ectopic lesions of model model rats was significantly increased, and the difference was statistically significant $(P<0.05)$, suggesting that ectopic endometrium a-SMA is highly expressed. After ELC treatment, the expression of a-SMA in ELC groups(high medium and low dose groups) were all reduced compared with model groups $(P<0.05)$ (Figure 6$)$. These results suggest that ELC may reduce the fibrosis process of ectopic lesions by inhibiting the expression of a-SMA in ectopic lesions of endometriosis model rats.

\section{RNA-sequencing analysis of endometriosis rats model characteristic and the treatment of ELC}

\section{The differentially expressed genes(DEGs) screening analysis}

We further analyzed the potential mechanism of ELeng Capsule by RNA transcriptome.The tissues of Model_Ecto and ELC_Ecto groups were used for the mRNA microarray assays. According to the results of the principal components analysis, there was no significant difference between the endometria of rats in the endometriosis model and ELC intervention groups. The results of the principal components analysis also showed that the rat endometriosis model were no obvious eutopic endometrial changes. These suggested that ELC may not affect the eutopic endometrium in endometriosis rat models. There were a total of 1461 DEGs in control_Eu versus model_Ecto groups, 557 DEGs in model_Ecto versus ELC_Ecto groups, and 1097 DEGs in control_Eu versus ELC_Ecto groups(FC-1.5). Supplementary material 5 showed the PCA of five groups,veen analysis of five group, up- and down regulated DEGs and Heatmap illustration. In this study, there were 1048 up-regulated DEGs and 413 down-regulated DEGs between Model_Ecto versus Con_Euto groups. The transcriptome analysis of the endometriosis rat model revealed some genes among the DEGs that participate in processes, such as inflammation, cytoskeleton, EMT, and angiogenesis. In the ELC_Ecto versus Model_Ecto group analysis, a total of 66 and 491 up-regulated and down-regulated DEGs, respectively, were identified, reflecting the differential expression of related genes after treatment with ELC. 
As shown in GO terms, the up-regulated genes were most significantly enriched in the CC of extracellular region, the BP of muscle contraction, and MF of actin filament binding, fibronectin binding, calcium ion binding, etc. The actin-associated GO terms may relate to the development of ectopic lesions in the endometriosis rat model(Figure 7A). The KEGG analysis of genes associated with endometriosis suggested that these genes are associated with the pathway of extracellular matrix-receptor (ECM-receptor) interaction, p53 signaling pathway, pathways in cancer, endocrine resistance, interleukin-17 (IL-17) signaling pathway, chemokine signaling pathway, cytokine-cytokine receptor interaction, etc(Figure 7B).

We performed a Venn analysis of DEGs and human endometriosis-related genes using Genecard datasets. The results showed that there were 113 upregulated and 28 down-regulated genes that were related to endometriosis. The proteins expressed by these genes are involved in EMT and may be involved in the process of fibrosis in endometriosis. The up-regulated genes associated with endometriosis in humans are related to cytokine-cytokine receptor interaction, phosphatidylinositol 3 kinase-Akt (PI3K-Akt) signaling pathway, pathway in cancer, MAPK signaling pathway, ECM-receptor interaction, Ras signaling pathway, toll-like receptor (TLR) signaling pathway, IL-17 signaling pathway, p53 signaling pathway, forkhead box protein 0 signaling pathway, focal adhesion, etc. Based on the above analyses, the rat model of endometriosis may be suitable for investigating the transcriptome level.

The enrichment score for this signaling pathway was positively correlated to endometriosis samples. Based on the path of GSEA enrichment[17], neuroactive ligand receptor interaction, cell adhesion molecules, and regulation of actin cytoskeleton are closely related to the occurrence and development of endometriosis. The development of endometriosis are related to the GO terms of "skeletal muscle fiber", "endodermal cell differentiation", "regulation of signaling receptor activity", "positive regulation of myoblast differentiation", "response to cytokine", "chemokine-mediated signaling pathway", "positive regulation of smooth muscle cell migration",etc. The KEGG pathways of GSEA showed that the peroxisome proliferator-activated receptor signaling, tumor necrosis factor signaling, MAPK signaling, apelin signaling, hypoxia-inducible factor-1 signaling, PI3K-Akt signaling pathway, and focal adhesion are related to endometriosis(Figures $7 \mathrm{C}) .(P<0.01, \mathrm{FDR}<0.25)$.

\section{GO and KEGG enrichment analysis of ELC_Ecto versus Model_Ecto}

Based on the $\mathrm{GO}$ analysis, the development and drug(ELC) regulation of endometriosis animal models are related to the regulation of troponin and the cytoskeleton. The BP, CC, and MF GO terms suggest muscle- and troponin-associated regulation,which could related to ELC treatment. The major enriched GO BPs were positive regulation of fast-twitch skeletal muscle fiber contraction, muscle contraction, striated muscle contraction, etc. The major enriched GO CCs were terminal cisterna, junctional sarcoplasmic reticulum membrane, Z disc, etc. The major enriched GO MFs were actin filament binding, structural constituent of muscle, actin binding, etc. (Figure 8A).

We also analyzed the KEGG pathways of the ELC ectopic endometrium(ELC_Ecto) versus the model ectopic endometrium(Model_Ecto). The results of KEGG pathway enrichment showed that down-regulated DEGs were mainly enriched in the following pathways: calcium signaling pathway, apelin signaling pathway, cyclic guanosine monophosphate-protein kinase G (cGMP-PKG) signaling pathway, 5 ' adenosine monophosphate-activated protein kinase signaling pathway, hypoxia-inducible factor-1 signaling pathway, MAPK signaling pathway, PI3K-Akt signaling pathway, focal adhesion, etc(Figure 8B).These pathways could be related to the inhibitory effect of ELC in endometriosis treatment.

Through the GSEA of the KEGG analysis, we also found other signaling pathways, including the Notch signaling pathway, adherens junction, Hippo signaling pathway, basal transcription factors, regulation of actin cytoskeleton, etc., which were related to the treatment of endometriosis with ELC.The The major KEGG pathways from GSEA analysis were shown in Figure $8 \mathrm{C}(\mathrm{FDR}<0.25)$. ELC may inhibit fibrosis and EMT by regulating the aforementioned pathways.

The core genes established in the network may be related to the regulation by ELeng Capsule treatment. Two modules obtained using the default criteria of MCODE for ELC_Ecto versus Model_Ecto were used to construct the network. We selected major modules for module network visualization (Figure 8D). The core nodes continued to be associated with genes related to actin, cytoskeleton, and fibrosis.

\section{STEM analysis of differential expression patterns}

Temporal expression patterns and the significance of the genes were determined using the STEM software. This approach was used to profile the DEGs, select significant gene clusters with parallel expression patterns, and identify the profiles of the "up to down" model from control to model to treatment with ELC. The results of the gene cluster analysis were statistically significant in profiles_14, profiles_11, profiles_10, and profiles_4 ( $P<0.05)$ (Figure 9A). After the development of endometriosis model, actin-associated DEGs in the Model_ecto and ELC_ecto groups were up-regulated; these may be the regulatory genes for the development of the endometriosis model (profile_11 and profile_4). The series test of profile_14 and profile_10 showed that the significant clusters were considered as potential profiles that could be affected by treatment with ELC $(P<0.05)$. Several actin-related and microfilament proteins were up-regulated in the model group and down-regulated after treatment, suggesting that the overall regulation mechanism of ELC treatment in ectopic endometriun is related to the regulation of actin cytoskeleton. The results of the STEM analysis were further reflecting the process of the endometriosis rat model and intervention with ELC(Figure 9B).

\section{Protein-protein interaction network}

We constructed network relationships between the core genes of the two groups of DEGs, and analyzed the possible network relationships through relevant pathways. We selected the calcium signaling pathway, cGMP-PKG signaling pathway, apelin signaling pathway, and the DEGs to construct the network (Figure 10A). The hub down-regulated genes closely related to treatment with ELC were as follows: Actn3, Actn2, Myom2, myoglobin, Ryr1, Myog, Myh7, Myod1, sarcalumenin, myosin light chain kinase 2, Smyd1, Map3k7, Mapk12, Myh4, Cacna1s, EEF1A2, and Cacng1. 


\section{CMAP analysis}

We compared the DEGs in gene expression profiles between induced by ELC(ELC_Ecto) vs Model_Ecto groups,and used the CMAP database to better understand the therapeutic efficacy and identify potential targets of this agent. Based on the results, a network was constructed to link ELC with representative drugs and their therapeutic targets. The profile of ELC was similar to that of classical pharmacotherapies (e.g.,sulfaphenazole, carbinoxamine, esculin, emetine, zoxazolamine, and niflumic acid). The result of CMap analysis are showed in Table 5. Carbinoxamine is a first-generation antihistamine of the ethanolamine class. Zoxazolamine is a centrally acting myorelaxant used as an antispasmodic and uricosuric. Niflumic acid are used in the treatment of rheumatoid arthritis, dysmenorrhea, and osteoarthritis. Niflumic acid inhibited invasion and reduced apoptosis in CNE-2Z cells through regulation of the extracellular regulatory kinase/MAPK pathway. Thus, this agent may serve as a candidate of anticancer drug [21, 22]. Based on the results of the CMAP analysis, we consider that ELC may similarly regulate inflammation, the cytochrome C P450 pathway, and MAPK pathway.

\section{Real-time quantitative RT-PCR}

Quantification was performed with a two-step reaction process: reverse transcription (RT) and PCR. The expression ratios of these DEGs, determined by qPCR, were consistent with those obtained from the mRNA transcriptome analysis(Figure 10B). The results for some genes exhibited a different trend; however, the differences were not statistically significant. The observed trend may be related to individual differences in the degree of gene expression in rats. The expression of verified genes in qPCR were showed in supplementary material 6.

\section{Discussion}

The mechanism of Traditional Chinese medicine in the treatment of endometriosis could related to inhibit inflammation, enhance the immune response, regulate angiogenesis-related pathways, and inducing apoptosis[23]. In this study, we identify potential molecular mechanisms involved network pharmacology and investigated the regulated genes of ELC directly associated with endometriosis rat models by RNA-sequencing.

\section{The characteristic of endometriosis rat model}

In this study, we found that up-regulated DEGs were significantly enriched in several pathways, including focal adhesion, ECM-receptor interaction, calcium signaling pathway, and cytokine-cytokine receptor interaction. Moreover, up-regulated DEGs may play important roles in the development of endometriosisassociated BP and pathways (e.g., inflammation, immunity, EMT, wound healing, and stem cell behavior), and contributes pathologically to fibrosis and progression of cancer.

EMT and fibroblast-to-myofibroblast transdifferentiation, as well as increases in cellular contractility, collagen production, and smooth muscle metaplasia lead to fibrosi[24, 25].A study investigating a rat model of endometriosis showed that the up-regulated genes included those encoding cytokines, chemokines, growth factors, and cell adhesion molecules.Endometriosis may be triggered by infection, mechanical damage, and inflammation, as each of these mechanisms can induce EMT in the mesothelium[26].In endometriosis, the endometrial stroma and glands exhibit ectopic growth and are surrounded by dense fibrous tissue[27]. The results of the transcriptome analysis in endometriosis model rats also suggested endometriosis are realated to EMT.

Endometriotic tissue is often induced in rodents via transplantation through surgery or intraperitoneal injection of uterine tissue fragments. The time of collection in rat models is 8 weeks after modeling; hence, the lesion has begun to undergo fibrosis. The interstitial space is a possible source of fibrotic cells during serosal inflammation and tissue repair.Therefore, it may play an important role in peritoneal fibrosis and adhesion formation[28]. Actin, a member of the ezrin-radixin-moesin family, plays a role in cell movement by linking the actin cytoskeleton. The roles of actin and the cytoskeleton in the development of endometriosis, as well as the relationship with cell adhesion, invasion, and fibrosis, also attracted our attention[29].The result of RNA-seq showed that the rat models of endometriosis could represent the characteristics of endometriosis to a certain extent and could provide the basis for further pharmacological research[30].

\section{Potential mechanism of ELC treatment}

In this study,we had identified 40 coumpouds in ELC and established compounds and targets network, and further analysis of the potential mechanism involved in treatment with ELC by network pharmacology and RNA-sequence. Compounds in ELC could relieve endometriosis-associated pain and regulated the neuroactive ligand-receptor interaction, metabolism of xenobiotics by cytochrome P450, TLR signaling, VEGF signaling, calcium signaling pathway.We further analyze the mechanism of the core compounds in ELC.

The reported efficacy of servical compounds are related to the mechanism of ELC in endometriosis treatment.The coupoumds of Curcuma phaeocaulis and Sparganium stoloniferum are common compounds with anti-cancer properties. Sparstolonin B could serve as a potential therapeutic agent for the treatment of TLR-mediated inflammatory disorders[31],also alleviate neuropathic pain by selectively suppressing TLR2 and TLR4[32]. $\beta$-elemene possesses broadspectrum antitumor activity and is effective against several types of tumors[33]. The active ingredients of Salvia miltiorrhiza include tanshinone I, tanshinone IIA, salvianolic acid, dihydrotanshinone, etc[34]. Tanshinone IIA could reduce the VEGF/VEGFR2 pathway and CD146 in vitro and in vivo, and regulates angiogenic function in human umbilical vein endothelial cells [35]. Rosmarinic acid is a potential natural compound with anti-cancer properties, as demonstrated in various human cancer cell lines[36]. It also could inhibit the proliferation of primary and T-HESCs and induce cell cycle arrest of the latter in the G2/M phase in vitro[37]. Paeonia lactifora has hematopoietic functions, anti-inflammatory activity, and immunological properties.In $P$. lactifora, paeoniflorin could inhibit the plantar incision-induced microglia TLR4/MMP-9/2/IL-1 $\beta$ signaling pathway and suppresses postoperative pain[38]. Paeoniflorigenone could induce apoptosis and exerts an antiproliferative effect[39]. Citrus aurantium may possess anti-tumor activity. Naringenin could induce apoptosis and endoplasmic reticulum stress through regulation of the MAPK and Akt signal transduction pathways in End1/E6E7 and VK2/E6E7 cells[40]. Limonin could induce apoptosis, thereby affecting the growth of SNU449 and HCT-15 tumor cells[41]. These small molecule compounds may exert 
new synergistic regulatory effects. The mechanism in endometriosis treatment of above compounds need to be futher studied. ELC have the characteristics of multi-target regulation, which may regulate angiogenesis and induce apoptosis based on network pharmacology and experimental verification.

In order to further explore the regulatory mechanism of ELC in the transcription level, we further conducted mRNA transcriptomics analysis. The transcriptome results showed that ELC treatment could relieve endometriosis through regulating the biological processc of ytoskeleton, troponin, EMT,etc. After the intervention with ELC, the expression of genes related to troponin,cytoskeleton and the MAPK signaling pathwaywere down-regulated compared with that measured in the model group[42].The results of the GSEA suggest that the formation of a rat model of endometriosis and the intervention of ELC on endometriosis model rats are related to the cytoskeleton, EMT, fibrosis, and muscle fibrosis, which are more closely related to abdominal endometriosis and deep infiltration of endometriosis patients. And the result of Masson's trichrome staining and a-SMA expression also identified the inhibition effection of fibrosis of ELC.In addition, through the GSEA of the ELC_Ecto group and the Model_Ecto group, the results of the KEGG enrichment analysis revealed a relationship with the Notch signaling pathway and the Hippo signaling pathway. The hyperactivation of the ADAM17/Notch signaling pathway could result in an increase in fibrosis, which is associated with deep infiltrating endometriosis[43].

In other enriched signaling pathways of ELC regulation, 19 down-regulated DEGs were enriched in the calcium signaling pathway. Apelin,as a ligand of the APJ receptor,has functions in angiogenesis and cell proliferation, and is a vasoactive and regulatory peptide[44]. And apelin expression in the ectopic and ectopic endometrium changes periodicall[45].The DEGs in the Apelin signaling pathway are related to muscle contraction, calmodulin binding, and the myosin complex. Moreover,the kinase associated pathways are associated with endometriosis.A genome-wide association study analysis revealed that multiple pathways, new variants in MAP3K4, and several pathways linked to MAPK are associated with endometriosis[46]. The serine/threonine kinase Akt and extracellular regulatory kinase signaling pathways can synergistically support deep endometriosis by enhancing the proliferation and survival of endometrial stromal cells (ESCs) in the in vitro fibrotic microenvironment [47]. These above pathways, as related pathways for endometriosis, may participate in the regulation process of ELC.

Further,the DEGs were verified through qPCR. These genes may be involved in the intervention with ELC. Alterations in key pathways (MAPK pathway) regulating cell cycle checkpoints, apoptosis, and EMT, appear to be closely associated to the development of chemoresistance in cancer. These genes, which are related to tumors, cytoskeleton, and cell potential, have numerous biological functions and may be involved in the development of endometriosis. EEF1A2 encodes an isoform of the alpha subunit of the elongation factor-1 complex, and may be critical in the development of ovarian cancer[48]. Targeting EEF1A2 and plitidepsin to release protein kinase R may trigger the extrinsic pathway of MAPK and nuclear factor-kB-dependent activation, leading to tumor cell death[49]. RYR1 is the core factor of the calcium signaling pathway. The ryanodine receptor calcium release channel is central to the cytoplasmic calcium signaling pathway [50].

Based on this research, we also have new discovery about the mechanism of action of Chinese medicine for removing blood stasis.At present, current research on the role of TCM in promoting blood circulation and removing phlegm is focused on apoptosis, inflammatory immunity, angiogenesis in endometriosis. These results of proteomic studies suggest that endometriosis is associated with EMT, and that there are differences in differentially expressed proteins among various syndromes in TCM[51]. The mechanism for the regulation of cytoskeleton and EMT through TCM is lacking. Several natural compounds suggested that treatment in cancer, inflammatory and fibrosing diseases through the regulation of EMT process[52].

\section{Limitations}

There are several limitations in this study. Firstly, only the small molecule compounds derived from plants in ELC were analyzed in the UPLC-Q-TOF/MS analysis,without the source animals were not analyzed. Secondly, although we have shown that rats/mice are a good animal model for studying endometriosis, they cannot reflect the natural course of the human disease. Further research on cells is warranted to clarify the mechanism involved in the intervention with ELC. Third, the relationship between the regulation of the cytoskeleton and troponin and the presence of endometriosis, and further optimization of the core components of ELC.

\section{Conclusions}

In this study, we have explained the treatment mechanism of ELeng capsules using transcriptome analysis and network pharmacology. According to the transcriptomic and network pharmacology analyses, we hypothesized that ELC may regulate inflammation and immunity, regulate cell adhesion, cytoskeletonrelated genes, influence the process of EMT, and consequently affect the development of lesions. The use of network pharmacology combined with transcriptome could help us understand the associated targets and pathways networks, candidate genes in ELeng Capsule. Combined techniques may also offer an efficient method of drug discovery in herbal medicines.

\section{Abbreviations}

ADMET, absorption, distribution, metabolism, excretion and toxicity

BP: biological process

CC: cellular component

DEGs,differentially expressed genes

DL,drug-likeness 
GO: gene ontology

KEGG:Kyoto Encyclopedia of Genes of Genomes

MCODE: Molecular Complex Detection

MF: molecular function

OB:oral bioavailability

PCA:principle component analysis

PPI: protein-protein interactions

QC:quality control

RT-PCR:real time polymerase chain reaction

TCMSP: Traditional Chinese Medicine Systems Pharmacology

UPLC-Q-TOF/MS:ultraperformance liquid chromatography with quadrupole time-of flight mass spectrometry

\section{Declarations}

\section{Ethics approval and consent to participate}

This study was carried out in accordance with the recommendations of the Guidelines for the Care and Use of Laboratory. Animals, and the protocols of animal breeding, handling and surgical protocolswere approved by the Guangdong Provincial Hospital of Chinese Medicine Committee on the Use of Live Animals for Teaching and Research (SZY2016007).

\section{Consent for publication}

Not applicable.

\section{Availability of data and materials}

All data is contained within the manuscript and additional files.

The entire RNA-seq dataset is available at the sequencing Read Archive (SRA) database under the accession

number SRR10357956,SRR10357949,SRR10357964,

SRR10357959,SRR10357963,SRR10357945,SRR10357958,SRR10357965,SRR10357953,SRR10357946,SRR10357947,SRR10357952,SRR10357948,SRR103

SRR10357961,SRR10357957,SRR10357962,SRR10357950,SRR10357951,SRR10357954,SRR10357960.

(https://dataview.ncbi.nlm.nih.gov/object/PRJNA579050).

\section{Competing interests}

The authors declare that they have no competing interests.

\section{Funding}

This study is supported by the National Natural Science Foundation of China (81574008), Special Research Project for Traditional Chinese Medicine of Guangdong Hospital of TCM (NO.YN2016ML05). The funder (Lixing Cao) provided important supports during design of the study and collection, analysis, and interpretation of data and in writing the manuscript.

\section{Authors' contributions}

All authors were responsible for the study concept and design: WZ drafted the paper. JW help drafted the paper. WZ,JW,JYW,YXH and TW participated in animal experiments.WZ and JW analysis of transcriptome results and disease models was performed.WZ and JG participated in network pharmacology analysis. LC and XL designed and supervised the study. WZ and JW have contributed equally for this work. The author(s) read and approved the final manuscript.

\section{Corresponding author}

Correspondence to Xuefang Liang and Lixing Cao.

\section{Acknowledgements}


The authors thank the whole team for assistance.

\section{References}

1. Dunselman GA, Vermeulen N, Becker C, Calhaz-Jorge C, D'Hooghe T, De Bie B, Heikinheimo O, Horne AW, Kiesel L, Nap A et al: ESHRE guideline: management of women with endometriosis. HUM REPROD 2014, 29(3):400-412.

2. Morotti M, Vincent K, Becker CM: Mechanisms of pain in endometriosis. Eur J Obstet Gynecol Reprod Biol 2017, 209:8-13.

3. Khan MA, Sengupta J, Mittal S, Ghosh D: Genome-wide expressions in autologous eutopic and ectopic endometrium of fertile women with endometriosis. Reprod Biol Endocrinol 2012, 10:84.

4. Flower A, Liu JP, Lewith G, Little P, Li Q: Chinese herbal medicine for endometriosis. Cochrane Database Syst Rev 2012(5):D6568.

5. Huang Yanghui CLSY: Effet of ELeng capulse on inhibit MMP/TIMP-1 in endometriosis-associated infertility. LIAONING JOURNAL OF TRADITIONAL CHINESE MEDICINE 2008, 35(5):658-660.

6. Xu MT LXCL: Effect and mechanism of Eleng capsule on pelvic cavity state in patients with pelvic endometriosis. Guangdong Medical Journal 2010(19):2589-2591.

7. Rogers PA, Donoghue JF, Walter LM, Girling JE: Endometrial angiogenesis, vascular maturation, and lymphangiogenesis. REPROD SCI 2009, 16(2):147151.

8. Mihalyi A, Simsa P, Mutinda KC, Meuleman C, Mwenda JM, D'Hooghe TM: Emerging drugs in endometriosis. Expert Opin Emerg Drugs 2006, 11(3):503524.

9. Zeng L, Yang K, Liu H, Zhang G: A network pharmacology approach to investigate the pharmacological effects of Guizhi Fuling Wan on uterine fibroids. EXP THER MED 2017, 14(5):4697-4710.

10. Zhang Y, Ye T, Gong S, Hong Z, Zhou X, Liu H, Qu H, Qian J: RNA-sequencing based bone marrow cell transcriptome analysis reveals the potential mechanisms of E'jiao against blood-deficiency in mice. BIOMED PHARMACOTHER 2019, 118:109291.

11. Duan X, Han L, Peng D, Chen W, Peng C, Xiao L, Bao Q: High Throughput mRNA Sequencing Reveals Potential Therapeutic Targets of Tao-Hong-Si-Wu Decoction in Experimental Middle Cerebral Artery Occlusion. FRONT PHARMACOL 2018, 9:1570.

12. Prather GR, MacLean JN, Shi M, Boadu DK, Paquet M, Hayashi K: Niclosamide As a Potential Nonsteroidal Therapy for Endometriosis That Preserves Reproductive Function in an Experimental Mouse Model. BIOL REPROD 2016, 95(4):76.

13. Gu J, Gui Y, Chen L, Yuan G, Lu HZ, Xu X: Use of natural products as chemical library for drug discovery and network pharmacology. PLOS ONE 2013, 8(4):e62839.

14. Shannon P, Markiel A, Ozier O, Baliga NS, Wang JT, Ramage D, Amin N, Schwikowski B, Ideker T: Cytoscape: a software environment for integrated models of biomolecular interaction networks. GENOME RES 2003, 13(11):2498-2504.

15. Vernon MW, Wilson EA: Studies on the surgical induction of endometriosis in the rat. FERTIL STERIL 1985, 44(5):684-694.

16. Bolger AM, Lohse M, Usadel B: Trimmomatic: a flexible trimmer for Illumina sequence data. BIOINFORMATICS 2014, 30(15):2114-2120.

17. Subramanian A, Tamayo P, Mootha VK, Mukherjee S, Ebert BL, Gillette MA, Paulovich A, Pomeroy SL, Golub TR, Lander ES et al: Gene set enrichment analysis: a knowledge-based approach for interpreting genome-wide expression profiles. Proc Natl Acad Sci U S A 2005, 102(43):15545-15550.

18. Ernst J, Bar-Joseph Z: STEM: a tool for the analysis of short time series gene expression data. BMC BIOINFORMATICS 2006, 7:191.

19. Warde-Farley D, Donaldson SL, Comes O, Zuberi K, Badrawi R, Chao P, Franz M, Grouios C, Kazi F, Lopes CT et al: The GeneMANIA prediction server: biological network integration for gene prioritization and predicting gene function. NUCLEIC ACIDS RES 2010, 38(Web Server issue):W214-W220.

20. Lamb J: The Connectivity Map: a new tool for biomedical research. NAT REV CANCER 2007, 7(1):54-60.

21. Chi Y, Li K, Yan Q, Koizumi S, Shi L, Takahashi S, Zhu Y, Matsue H, Takeda M, Kitamura M et al: Nonsteroidal anti-inflammatory drug flufenamic acid is a potent activator of AMP-activated protein kinase. J PHARMACOL EXP THER 2011, 339(1):257-266.

22. Luo S, Huang G, Wang Z, Wan Z, Chen H, Liao D, Chen C, Li H, Li B, Chen L et al: Niflumic acid exhibits anti-tumor activity in nasopharyngeal carcinoma cells through affecting the expression of ERK1/2 and the activity of MMP2 and MMP9. Int J Clin Exp Patho/ 2015, 8(9):9990-10001.

23. Weisheng B, Nezhat CH, Huang GF, Mao YQ, Sidell N, Huang RP: Discovering endometriosis biomarkers with multiplex cytokine arrays. Clin Proteomics $2019,16: 28$.

24. Zhang Q, Duan J, Olson M, Fazleabas A, Guo SW: Cellular Changes Consistent With Epithelial-Mesenchymal Transition and Fibroblast-to-Myofibroblast Transdifferentiation in the Progression of Experimental Endometriosis in Baboons. REPROD SCI 2016, 23(10):1409-1421.

25. Liu X, Zhang Q, Guo SW: Histological and Immunohistochemical Characterization of the Similarity and Difference Between Ovarian Endometriomas and Deep Infiltrating Endometriosis. REPROD SCI 2018, 25(3):329-340.

26. Albertsen HM, Ward K: Genes Linked to Endometriosis by GWAS Are Integral to Cytoskeleton Regulation and Suggests That Mesothelial Barrier Homeostasis Is a Factor in the Pathogenesis of Endometriosis. REPROD SCI 2017, 24(6):803-811.

27. Li J, Dai Y, Zhu H, Jiang Y, Zhang S: Endometriotic mesenchymal stem cells significantly promote fibrogenesis in ovarian endometrioma through the Wnt/beta-catenin pathway by paracrine production of TGF-beta1 and Wnt1. HUM REPROD 2016, 31(6):1224-1235.

28. Bruner-Tran KL, Mokshagundam S, Herington JL, Ding T, Osteen KG: Rodent Models of Experimental Endometriosis: Identifying Mechanisms of Disease and Therapeutic Targets. Curr Womens Health Rev 2018, 14(2):173-188.

29. Zhan H, Ma J, Ruan F, Bedaiwy MA, Peng B, Wu R, Lin J: Elevated phosphatase of regenerating liver 3 (PRL-3) promotes cytoskeleton reorganization, cell migration and invasion in endometrial stromal cells from endometrioma. HUM REPROD 2016, 31(4):723-733.

Page 12/26 
30. Gu ZY, Jia SZ, Leng JH: Establishment of endometriotic models: the past and future. Chin Med J (Engl) 2020.

31. Yepuri N, Dhawan R, Cooney M, Pruekprasert N, Meng Q, Cooney RN: Sparstolonin B: A Unique Anti-Inflammatory Agent. SHOCK 2019.

32. Jin G, Jin X, Zhou S: Sparstolonin B selectively suppresses tollike receptor2 and 4 to alleviate neuropathic pain. MOL MED REP 2018, 17(1):1247-1252.

33. Bi YH, Zhang LH, Chen SJ, Ling QZ: Antitumor Mechanisms of Curcumae Rhizoma Based on Network Pharmacology. Evid Based Complement Alternat Med 2018, 2018:4509892.

34. MEIm XD, Cao YF, Che YY, Li J, Shang ZP, Zhao WJ, Qiao YJ, Zhang JY: Danshen: a phytochemical and pharmacological overview. Chin J Nat Med 2019, 17(1):59-80.

35. Zhang Q, Liu X, Guo S: Progressive development of endometriosis and its hindrance by anti-platelet treatment in mice with induced endometriosis. REPROD BIOMED ONLINE 2016, 34(2):124-136.

36. Yesil-Celiktas O, Sevimli C, Bedir E, Vardar-Sukan F: Inhibitory effects of rosemary extracts, carnosic acid and rosmarinic acid on the growth of various human cancer cell lines. Plant Foods Hum Nutr 2010, 65(2):158-163.

37. Ferella L, Baston JI, Bilotas MA, Singla JJ, Gonzalez AM, Olivares CN, Meresman GF: Active compounds present inRosmarinus officinalis leaves andScutellaria baicalensis root evaluated as new therapeutic agents for endometriosis. REPROD BIOMED ONLINE 2018, 37(6):769-782.

38. Fan YX, Hu L, Zhu SH, Han Y, Liu WT, Yang YJ, Li QP: Paeoniflorin attenuates postoperative pain by suppressing Matrix Metalloproteinase-9/2 in mice. EUR J PAIN 2018, 22(2):272-281.

39. Huang Y, Ohno O, Suenaga K, Miyamoto K: Apoptosis-inducing activity and antiproliferative effect of Paeoniflorigenone from moutan cortex. Biosci Biotechnol Biochem 2017, 81(6):1106-1113.

40. Park S, Lim W, Bazer FW, Song G: Naringenin induces mitochondria-mediated apoptosis and endoplasmic reticulum stress by regulating MAPK and AKT signal transduction pathways in endometriosis cells. MOL HUM REPROD 2017, 23(12):842-854.

41. Rahman A, Siddiqui SA, Jakhar R, Kang SC: Growth inhibition of various human cancer cell lines by imperatorin and limonin from poncirus trifoliata rafin. Seeds. Anticancer Agents Med Chem 2015, 15(2):236-241.

42. Ping S, Ma C, Liu P, Yang L, Yang X, Wu Q, Zhao X, Gong B: Molecular mechanisms underlying endometriosis pathogenesis revealed by bioinformatics analysis of microarray data. ARCH GYNECOL OBSTET 2016, 293(4):797-804.

43. Gonzalez-Foruria I, Santulli P, Chouzenoux S, Carmona F, Chapron C, Batteux F: Dysregulation of the ADAM17/Notch signalling pathways in endometriosis: from oxidative stress to fibrosis. MOL HUM REPROD 2017, 23(7):488-499.

44. Luo X, Liu J, Zhou H, Chen L: Apelin/APJ system: A critical regulator of vascular smooth muscle cell. J CELL PHYSIOL 2018, 233(7):5180-5188.

45. Ozkan ZS, Cilgin H, Simsek M, Cobanoglu B, Ilhan N: Investigation of apelin expression in endometriosis. J Reprod Infertil 2013, 14(2):50-55.

46. Uimari O, Rahmioglu N, Nyholt DR, Vincent K, Missmer SA, Becker C, Morris AP, Montgomery GW, Zondervan KT: Genome-wide genetic analyses highlight mitogen-activated protein kinase (MAPK) signaling in the pathogenesis of endometriosis. HUM REPROD 2017, 32(4):780-793.

47. Matsuzaki S, Darcha C: Co-operation between the AKT and ERK signaling pathways may support growth of deep endometriosis in a fibrotic microenvironment in vitro. HUM REPROD 2015, 30(7):1606-1616.

48. Worley MJ, Liu S, Hua Y, Kwok JS, Samuel A, Hou L, Shoni M, Lu S, Sandberg EM, Keryan A et al: Molecular changes in endometriosis-associated ovarian clear cell carcinoma. EUR J CANCER 2015, 51(13):1831-1842.

49. Losada A, Munoz-Alonso MJ, Martinez-Diez M, Gago F, Dominguez JM, Martinez-Leal JF, Galmarini CM: Binding of eEF1A2 to the RNA-dependent protein kinase PKR modulates its activity and promotes tumour cell survival. Br J Cancer 2018, 119(11):1410-1420.

50. Dulhunty AF, Beard NA, Casarotto MG: Recent advances in understanding the ryanodine receptor calcium release channels and their role in calcium signalling. F1000Res 2018, 7.

51. Wen Y, Wang Y, Feng TT, Wei SB: Differential Proteomics Analysis of Endometriosis in Blood Stasis Syndrome. CHIN J INTEGR MED 2018, 24(12):925929.

52. Avila-Carrasco L, Majano P, Sánchez-Toméro JA, Selgas R, López-Cabrera M, Aguilera A, González MG: Natural Plants Compounds as Modulators of Epithelial-to-Mesenchymal Transition. FRONT PHARMACOL 2019, 10:715.

\section{Tables}

\section{Table 1 The major herbs of ELeng Capsule}




\begin{tabular}{|c|c|}
\hline Herb & Component \\
\hline $\begin{array}{l}\text { Curcuma phaeocaulis } \\
\text { (E'zhu) }\end{array}$ & Ginger plant, Wen Yujin Curcuma wenyujin Y.H. Chen et C. Ling, rhizome. \\
\hline $\begin{array}{l}\text { Sparganium stoloniferum } \\
\text { (Sanleng } \rrbracket\end{array}$ & Black-triangular plant black-triangular, Sparganium stoloniferum Buch.-Ham, tubers. \\
\hline $\begin{array}{l}\text { Slavia miltiorrhiza } \\
\text { (Danshen) }\end{array}$ & Salvia miltiorrhiza, Salvia .miltiorrhiza Bge, dry roots and rhizomes \\
\hline $\begin{array}{l}\text { Hirudo nipponica } \\
\text { (Shuizhi) }\end{array}$ & Mink animal otter, Hirudo nipponica Whitman, dry body. \\
\hline $\begin{array}{l}\text { Trionyx sinensis } \\
\text { (Biejia) }\end{array}$ & Cyprinidae, Trionyx sinensis Wiegmann, carapace \\
\hline $\begin{array}{l}\text { Paeonia lactifora Pall. } \\
\text { (Chishao) }\end{array}$ & Ranunculaceae, peony, Paeonia lactiflora pall, root \\
\hline $\begin{array}{l}\text { Citrus aurantium ( } \\
\text { Zhike) }\end{array}$ & Citrus aurantium L., a dried, immature fruit of the cultivar. \\
\hline Angelica laxifoliata (Danggui) & Angelica laxifoliatalroot. \\
\hline
\end{tabular}

\section{Table 2 Chromatographic conditions}

1.Flow phase gradient

\begin{tabular}{|lll|}
\hline Time $(\mathrm{min})$ & Flow Rate $(\mathrm{mL} / \mathrm{min})$ & $\% \mathrm{~B}$ \\
\hline 0 & 0.3 & 5 \\
\hline 3 & 0.3 & 5 \\
\hline 11 & 0.3 & 14 \\
36 & 0.3 & 95 \\
\hline 38 & 0.3 & 95 \\
\hline 38.1 & 0.3 & 5 \\
\hline 41 & 0.3 & 5 \\
\hline
\end{tabular}

2.MS parameter

\begin{tabular}{|ll|}
\hline parameter & value \\
\hline TOF mass range & $50 \rrbracket 1500$ \\
\hline lon Source Gas 1 & 50 \\
\hline Ion Source Gas 2 & 50 \\
\hline Curtain Gas & 35 \\
\hline Ion Spray Voltage Floating $(\mathrm{kV})$ & $-4500 / 5000$ \\
\hline lon Source Temperature $\left({ }^{\circ} \mathrm{C}\right)$ & 500 \\
\hline Declustering Potential & 100 \\
\hline Collision Energy & 10 \\
\hline
\end{tabular}

3.MS/MS parameter 


\begin{tabular}{|ll|}
\hline parameter & value \\
\hline Declustering Potential & 100 \\
\hline Collision Energy & \pm 40 \\
\hline Collision Energy Spread & 20 \\
\hline Ion Release Delay & 30 \\
\hline Ion Release Width & 15 \\
\hline
\end{tabular}

Table 3 Primers sequences used for real-time PCR reactions

\begin{tabular}{|llll|}
\hline No. & Gene Symbol & Forward primer & Reverse primer \\
\hline 1 & Myog & CGACCTGATGGAGCTGTA & GGTGGACAGGAAGGTAGT \\
\hline 2 & Smyd1 & ACCGTCTATTTAACAAGGAAGC & GCACCGTGGCATTTACTA \\
\hline 3 & Six1 & ATTAGTGAGGGAAACAAGTGC & GTTTGTTGCGTTACTAACATCG \\
\hline 4 & Cacna1s & CACCTGGTTCACCAACTTTAT & CTGATTCCTCATGGAGTCG \\
\hline 5 & Eef1a2 & CCAGCAAATACCCTCAACC & GTCTTCTCCTTGCCCATTC \\
\hline 6 & Ryr1 & AGCCGTATGTACCTGAGT & GTGGCGTCTTCCTGTAATC \\
\hline 7 & Actn2 & CCAGCGCCATGAATCAGATA & CTCCTCCTGGATCATGTACTC \\
\hline 8 & Myod1 & GACAGCAGGTGTGCATTC & TAGTAGCTCCATGTCCCAGT \\
\hline 9 & Mapk12 & CAGTGGACATTTGGTCTGTTG & TGGTCCAGGTGGTCATTG \\
\hline 10 & Myh4 & CAAGGTGAAGAACGCCTA & TCCAGCTCGTGGATATGC \\
\hline 11 & ACTB & GCGAGTACAACCTTCTTGC & TATCGTCATCCATGGCGAAC \\
\hline
\end{tabular}

Table 4 The result of compounds identification in ELeng Capsules 


\begin{tabular}{|c|c|c|c|c|c|c|c|c|}
\hline No & $\begin{array}{l}\text { Time } \\
\text { \min } \rrbracket\end{array}$ & $\begin{array}{l}\text { Additive } \\
\text { ion }\end{array}$ & $\begin{array}{l}M / Z \\
\text { Actual } \\
\text { value }\end{array}$ & $\begin{array}{l}M / Z \\
\text { Theoretical } \\
\text { value }\end{array}$ & ppm & $\begin{array}{l}\text { Molecular } \\
\text { formula }\end{array}$ & Name & MS/MS spcetra \\
\hline 1 & 0.83 & {$[\mathrm{M}-\mathrm{H}]-$} & 341.1089 & 341.1089 & -0.1 & $\mathrm{C} 12 \mathrm{H} 22 \mathrm{O} 11$ & Sucrose & $341.1095,179.0560,89.0244$ \\
\hline 2 & 2.05 & {$[\mathrm{M}-\mathrm{H}]-$} & 169.015 & 169.0142 & 4.5 & C7H6O5 & Gallic acid & $169.0172,125.0240,79.0196$ \\
\hline 3 & 3.45 & [M-H]- & 197.0456 & 197.0455 & 0.3 & $\mathrm{C} 9 \mathrm{H} 1005$ & Danshensu & $179.0352,135.0459,123.0448,72.9917$ \\
\hline 4 & 8.8 & {$[\mathrm{M}-\mathrm{H}]-$} & 495.15 & 495.1508 & -1.6 & $\mathrm{C} 23 \mathrm{H} 28012$ & Oxypaeoniflora & $495.1503,465.1395,165.0558,137.0245,93.0340$ \\
\hline 5 & 12.47 & {$[\mathrm{M}+\mathrm{FA}]-$} & 525.1618 & 525.1614 & 0.8 & $\mathrm{C} 23 \mathrm{H} 28011$ & Paeoniflorin & $449.1444,327.1075,165.0551,121.0283$ \\
\hline 6 & 14.4 & {$[\mathrm{M}-\mathrm{H}]-$} & 595.1693 & 595.1704 & -1.8 & C27H32O15 & Neoeriocitrin & $595.1663,459.1138,339.0707,287.0549,235.0235,151.0$ \\
\hline 7 & 14.99 & {$[\mathrm{M}-\mathrm{H}]-$} & 579.1733 & 579.1719 & 2.4 & C27H32O14 & Narirutin & $579.1745,271.0605,151.0034$ \\
\hline 8 & 15.29 & {$[\mathrm{M}-\mathrm{H}]-$} & 579.1732 & 580.54 & 2.2 & C27H32014 & Naringin & $549.1717,459.1154,313.0710,271.0594,177.0183,151.0$ \\
\hline 9 & 15.77 & {$[\mathrm{M}-\mathrm{H}]-$} & 609.182 & 609.1825 & -0.8 & $\mathrm{C} 28 \mathrm{H} 34015$ & Neohesperidin & $609.1813,489.1405,343.0813,301.0694,286.0455,242.0$ \\
\hline 10 & 16.01 & {$[\mathrm{M}-\mathrm{H}]-$} & 359.0772 & 359.0772 & -0.1 & $\mathrm{C} 18 \mathrm{H} 1608$ & Rosmarinic acid & $197.0457,179.0349,161.0244,135.0450,133.0293,123.0$ \\
\hline 11 & 16.69 & {$[\mathrm{M}-\mathrm{H}]-$} & 717.1475 & 717.1461 & 1.9 & С36H30016 & Salvianolic acid B & $717.1460,519.0935,339.0507,321.0393,295.0610,279.0$ \\
\hline 12 & 17.09 & {$[\mathrm{M}-\mathrm{H}]-$} & 315.1605 & 315.1602 & 1 & $\mathrm{C} 19 \mathrm{H} 2404$ & $\begin{array}{l}\text { 1,7-bis(4- } \\
\text { hydroxyphenyl)-3,5- } \\
\text { heptanediol }\end{array}$ & $163.0748,149.0607,121.0656,106.0412,93.0340$ \\
\hline 13 & 17.3 & {$[\mathrm{M}-\mathrm{H}]-$} & 493.1135 & 493.114 & -1.1 & $\mathrm{C} 26 \mathrm{H} 22 \mathrm{O} 10$ & Salvianolic acid & $493.1152,313.0720,295.0612,185.0246,109.0290$ \\
\hline 14 & 18.17 & {$[\mathrm{M}-\mathrm{H}]-$} & 267.031 & 267.0299 & 4.1 & $\mathrm{C} 15 \mathrm{H} 805$ & Sparstolonin B & $267.0308,182.0425,167.0593,154.9976$ \\
\hline 15 & 19.45 & {$[\mathrm{M}-\mathrm{H}]-$} & 301.0715 & 301.0718 & -0.9 & $\mathrm{C} 16 \mathrm{H} 1406$ & Hesperetin & $301.0717,164.0118,151.0040,136.0169$ \\
\hline 16 & 19.46 & {$[\mathrm{M}-\mathrm{H}]-$} & 329.2337 & 329.2333 & 1.1 & C18H3405 & sanleng acid & $309.2328,229.1444,211.1342,193.1230,183.1389,171.1$ \\
\hline 17 & 21.65 & {$[\mathrm{M}-\mathrm{H}]-$} & 407.2816 & 407.2803 & 3.2 & $\mathrm{C} 24 \mathrm{H} 4005$ & Cholic acid & $407.2807,343.2621$ \\
\hline 18 & 21.72 & {$[\mathrm{M}+\mathrm{FA}]-$} & 515.1941 & 515.1923 & 3.5 & $\mathrm{C} 26 \mathrm{H} 3008$ & Limonin & $515.1942,469.1885,278.1320,229.1237$ \\
\hline 19 & 22.07 & {$[\mathrm{M}+\mathrm{H}]+$} & 237.1845 & 237.1849 & -1.7 & $\mathrm{C} 15 \mathrm{H} 2402$ & Curdione & $163.1117,137.0957,121.1012,107.0853,91.0540,79.536$ \\
\hline 20 & 27.38 & {$[\mathrm{M}+\mathrm{H}]+$} & 279.2323 & 279.2319 & 1.6 & C18H3002 & Linolenic acid & $295.2279,277.2176,195.1396,171.1031$ \\
\hline 21 & 27.71 & {$[\mathrm{M}+\mathrm{H}]+$} & 297.1483 & 297.1485 & -0.7 & $\mathrm{C} 19 \mathrm{H} 2003$ & Cryptotanshinone & $297.1479,179.1380,25.0930,251.1423,249.0908,139.07$ \\
\hline 22 & 27.71 & {$[\mathrm{M}-\mathrm{H}]-$} & 277.0863 & 277.0859 & 1.4 & $\mathrm{C} 18 \mathrm{H} 12 \mathrm{O} 3$ & Tanshinone I & $277.0854,249.0903,234.193 .0998,178.0770$ \\
\hline 23 & 28.94 & {$[\mathrm{M}+\mathrm{H}]+$} & 219.1742 & 219.1743 & -0.6 & $\mathrm{C} 15 \mathrm{H} 22 \mathrm{O}$ & $\begin{array}{l}\text { (E,E)-Germacra- } \\
3,7(11), 9 \text {-trien-6- } \\
\text { one }\end{array}$ & $159.1155,129.0692,105.0689,95.0847,91.0536,79.0532$ \\
\hline 24 & 30.19 & {$[\mathrm{M}+\mathrm{H}]+$} & 295.1335 & 293.1183 & 2.1 & C19H1803 & Tanshinone IIA & $295.1482,277.1377,213.0663,163.1134,151.1120,107.0$ \\
\hline 25 & 34.01 & {$[\mathrm{M}-\mathrm{H}]-$} & 279.2337 & 279.233 & 2.7 & C18H32O2 & Linoleic acid & Slavia miltiorrhiza \\
\hline
\end{tabular}




\section{Table 5 CMap analysis results}

\begin{tabular}{|lllllllll|}
\hline No. & Cmap name & Mean & $\mathbf{n}$ & Enrichment & $\boldsymbol{P}$ & Specificity & $\begin{array}{l}\text { Percent non- } \\
\text { null }\end{array}$ & Categories \\
\hline 1 & sulfaphenazole & 0.393 & 4 & 0.886 & 0.00016 & 0 & 50 & Amides,Antibacterials for Systemic Use, \\
& & & & & & & & Cytochrome P-450 CYP2B6 Inhibitors \\
\hline 2 & carbinoxamine & 0.447 & 4 & 0.781 & 0.0043 & 0 & 50 & Antihistamines for Systemic Use, \\
\hline 3 & esculin & 0.362 & 4 & 0.743 & 0.00824 & 0 & 50 & Cytochrome P-450 CYP3A Substrates \\
\hline 4 & emetine & 0.557 & 4 & 0.695 & 0.01818 & 0.2887 & 75 & bioflavanoid \\
\hline 5 & zoxazolamine & 0.427 & 4 & 0.639 & 0.04152 & 0.0351 & 50 & Anti-Infective Agents,Enzyme Inhibitors \\
\hline 6 & niflumic acid & 0.412 & 4 & 0.628 & 0.0479 & 0.0221 & 50 & acting myorelaxant \\
\hline & & & & & & & & Anti-Inflammatory Agents,Analgesics,Enzyme \\
Inhibitors, \\
Antiinflammatory and Antirheumatic Products
\end{tabular}

\section{Figures}

A
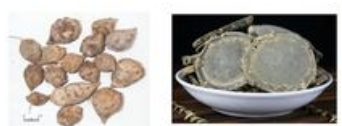

Sparganium stoloniferam Curcuma phaeocaulis

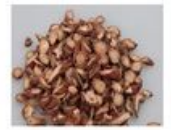

Slavia miltiorrhiza

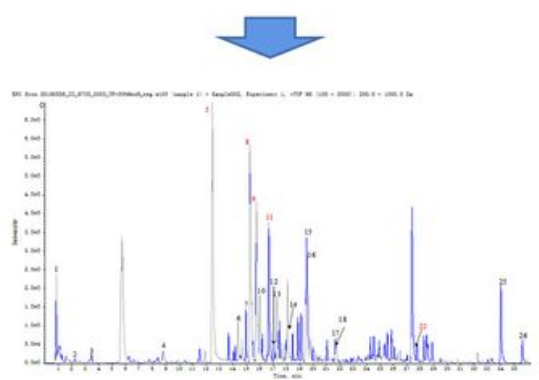

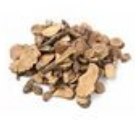

Curcuma wenyujim
Hirudo nipponica Trionyx sinemsis

Citrus aurantium

Angelica laxifoliata

Paeonia lactifora

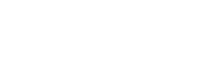

D
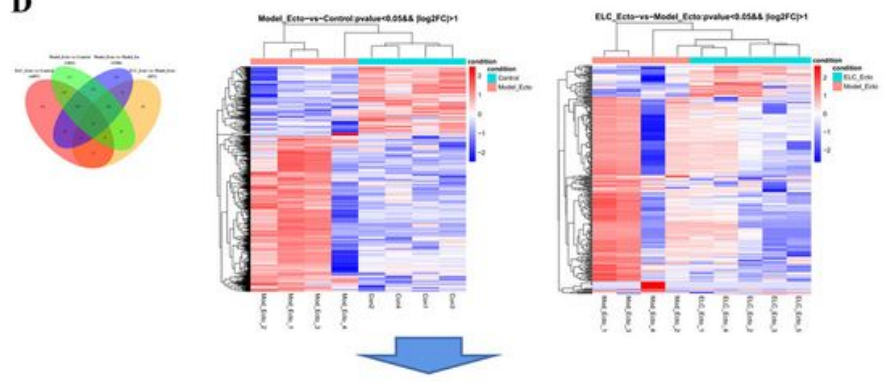

D

B

C
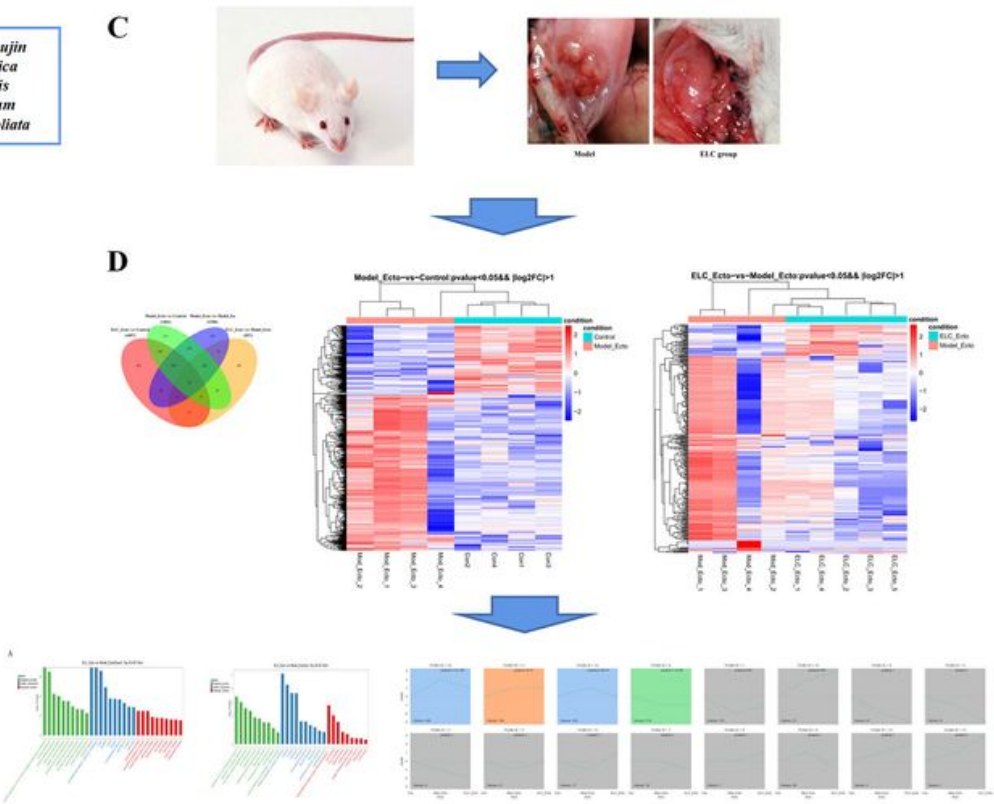
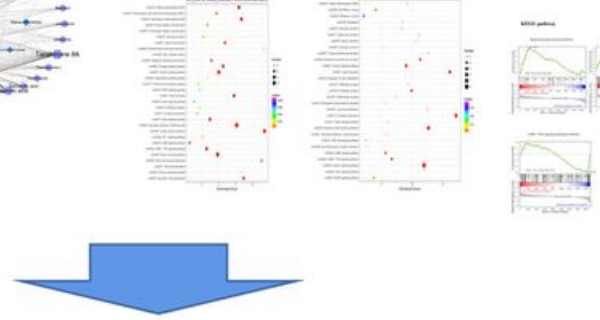

Multi-target regulation mechanism of ELeng Capsule 
The workflow of network pharmacology and RNA-sequencing approach.
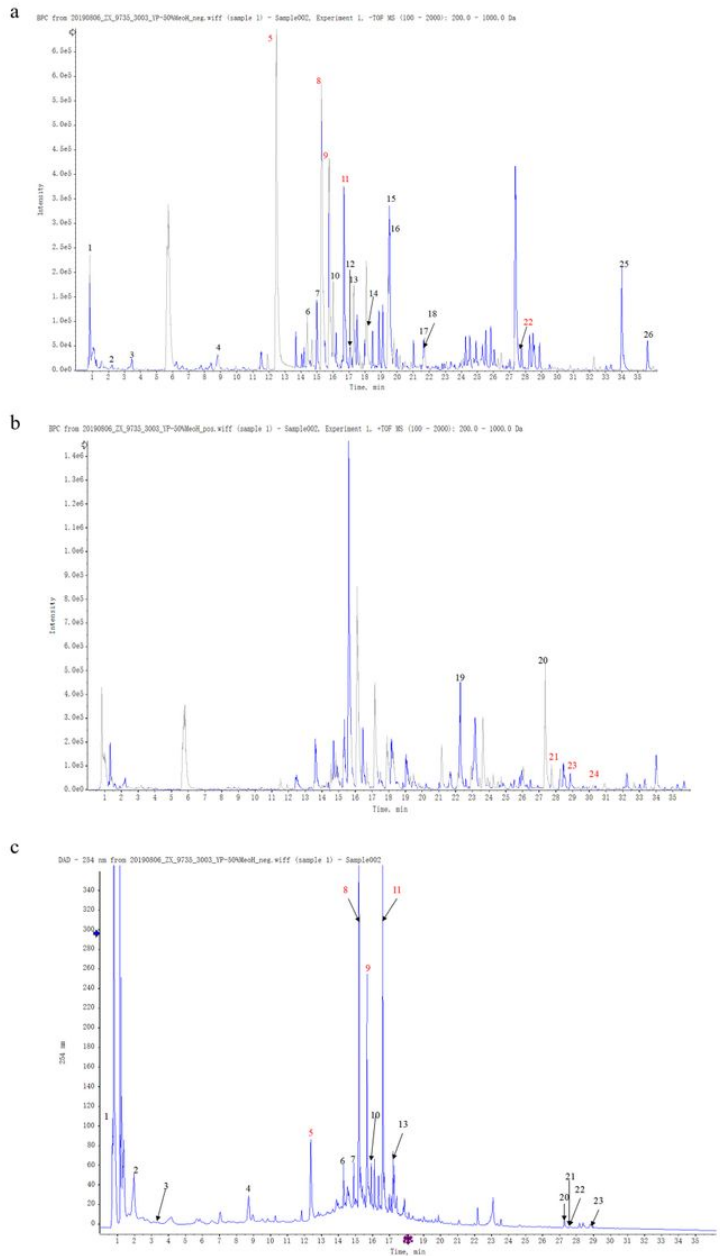

\section{Figure 2}

UPLC-TOF/MS analysis of ELeng Capsules (A) ELeng Capsules mass spectrometry negative ion mode identification chromatographic peak number diagram. (B) Identification of chromatographic peak numbers by mass spectrometry positive ion mode. (C) DAD spectrum (UV 254 nm). 1.Sucrose;2.Gallic acid;3.Danshensu;4.0xypaeoniflora;5.Paeoniflorin;6Neoeriocitrin; 7.Narirutin;8.Naringin;9.Neohesperidin;10.Rosmarinic acid;11.Salvianolic acid B;12.1,7-bis(4hydroxyphenyl)-3,5-heptanediol;13.Salvianolic acid;14.Sparstolonin B;15.Hesperetin;16.sanleng acid;17.Cholic acid;18.Limonin;19.Curdione;20.Linolenic acid;21.Cryptotanshinone;22.Tanshinone l;23.(E,E)-Germacra-3,7(11),9-trien-6-one;24.Tanshinone IIA;25.Linoleic acid;26.Palmitic acid.The table 4 showed the compouds identification. 


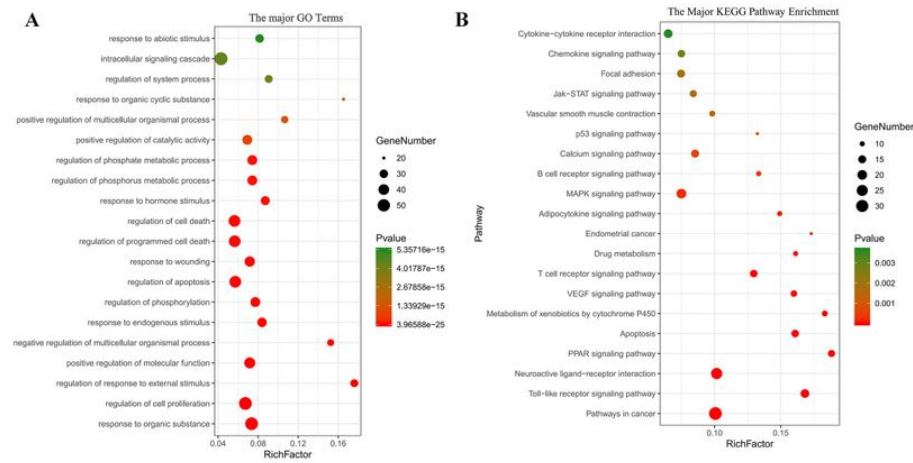

C

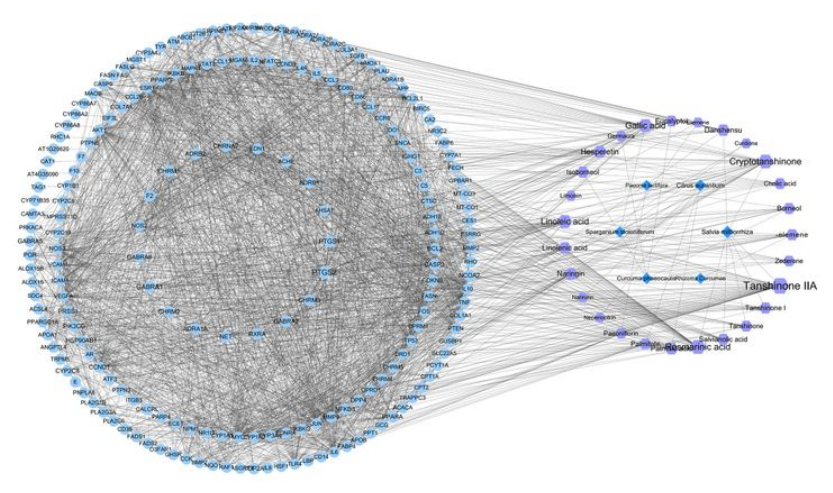

D

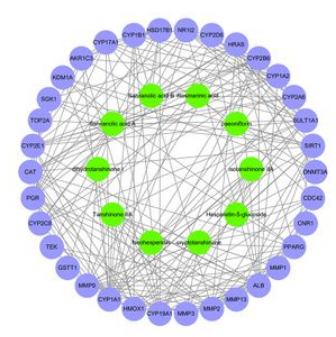

Figure 3

Network pharmacology analysis of ELeng Capsule. (A)The major GO terms of BP of potential targets of ELeng Capsule.(B)The major 20 KEGG pathways of potenial targets of ELeng Capsule. (C) Herb-compound-target-signaling pathway of ELC. The light blue nodes represent targets(genes); the purple nodes represent compounds; thedark blue nodes represent herbs.(D)The network of molecular docking between major compounds and targets. The purple nodes represent targets(genes); the green nodes represent compounds. 
A

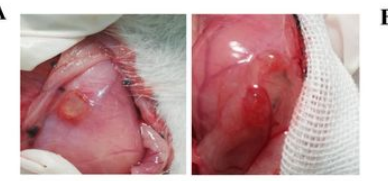

B
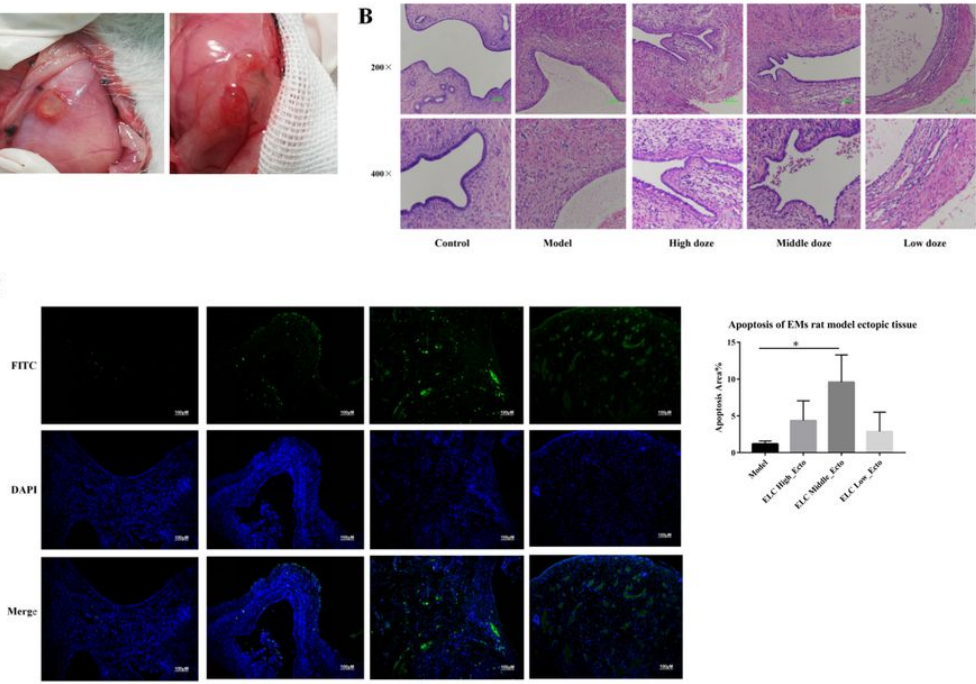

Mode__Ecto
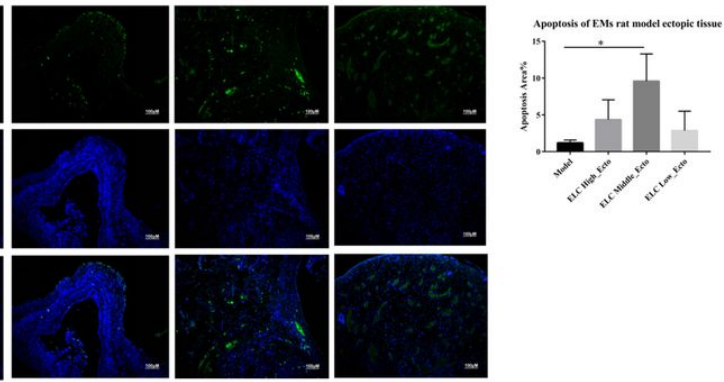

ELC midalle dose_ECET

EIC Chigh dore_Ecto

D

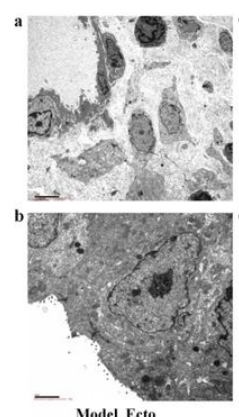

Model_Ecto
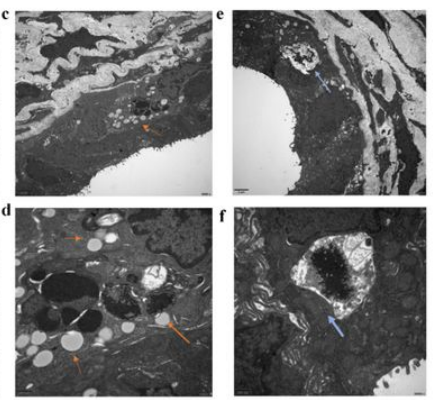

ELC_Ecto Middle doze

\section{Figure 4}

Pathology analysis. (A) Graphs of control sample excised from ectopic endometrium lesion in experimental rat. (B) The microstructure of ectopic endometrium. The effect of ELeng on the growth of endometriotic implants $(200 \times, 400 x)$. (C) ELeng Capsules Promoted Apoptosis in Ectopic Endometrial Tissues.Apoptosis was confirmed by TUNEL assay $(100 \mathrm{x}, \mathrm{n}=4)$. The green fluorescent signal represents the nucleus of a positively stained apoptotic cell. Compared with the model group, the apoptotic area in the ELC middle dose group was significantly increased $(P<0.05)$. (D)Ultrastructure of ectopic lesions. Ecto: Ectopic Endometrium;The blue arrow indicates autophagosome. The orange arrow shows the structure of apoptosis bodies. 


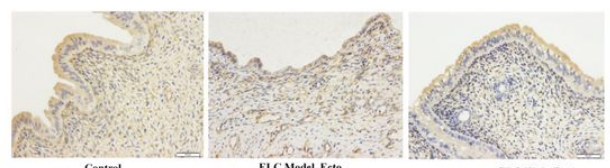

Eic Model_Ecto

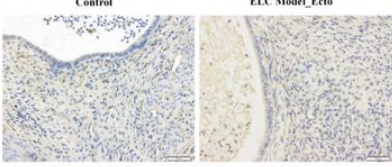

ELC High_Ect

ELC Middle_Ecto

ELC Low_Ecto

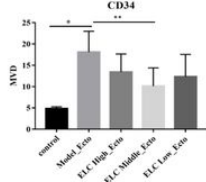

B

VEGFA

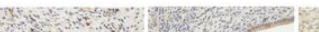

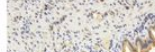

is sivena

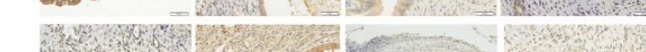

vEGB Nover

VEGFC

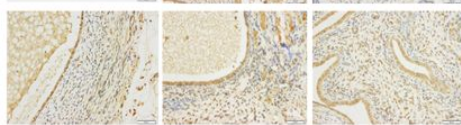

Model_Ecto

ELC High_Ecto

ELC Middle_Ecto

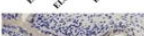

VEGFA
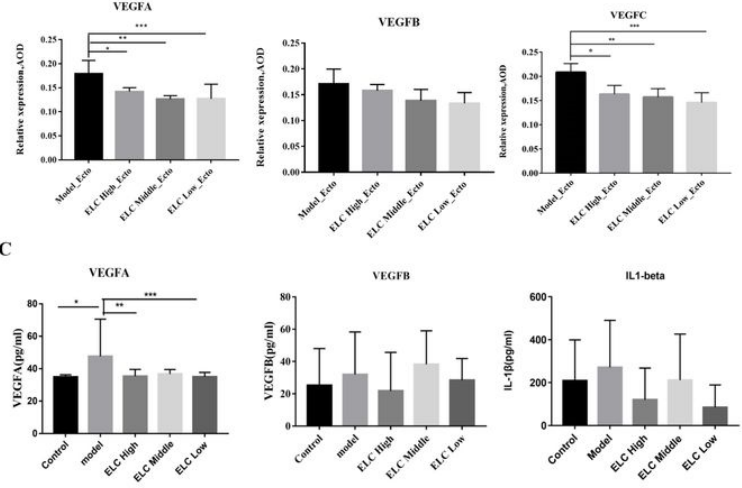

\section{Figure 5}

The result of angiogenesis expression in ectopic lesion in endometriosis model rats and VEGFA, VEGFB,IL1-ßexpression in serum Values are represented as mean $\pm S D, n=4$. $p<0.05$ as determined by one-way ANOVA analysis. (A)Compared with the control group eutopic endometrium,the MVD in ectopic endometrium in model group were increased $(n=6, * P=0.001<0.05)$. Compared with the model group,the MVD in ectopic endometrium in ELC middle dose were deseased $(* \star$ $P=0.018<0.05)$.(B) The results suggested that the expression of VEGFA were statistically significant $(P=0.014) . * P=0.031 \rrbracket 0.05, \star \star P=0.004 \llbracket 0.05, \star \star \star P=0.005 \rrbracket$ 0.05.There was no significant difference in different groups of VEGFB expression( $P>0.05)$.And compared with the modle group,the expression of VEGFC was reduced in ELC groups. ${ }^{\star} \mathrm{P}=.005,{ }^{*} \mathrm{P}=0.002, * \star * \mathrm{P}=0.000, \varangle 0.05$. (C) The expression levels of VEGFA in serum were statistically significant $(\mathrm{F}=2.742, \mathrm{P}=0.044<$ 0.05). Where, ${ }^{*}=0.008 ; * \star P=0.020 ; * \star * P=0.012$. There was no significant difference in different groups of the expression levels of $V E G F B$ in serum( $F=0.674$, $\mathrm{P}=0.614>0.05)$. There was no significant difference in different groups of the expression levels of IL $1 \beta$ - expression( $F=1.399 \mathrm{P}=0.255>0.05)$. 


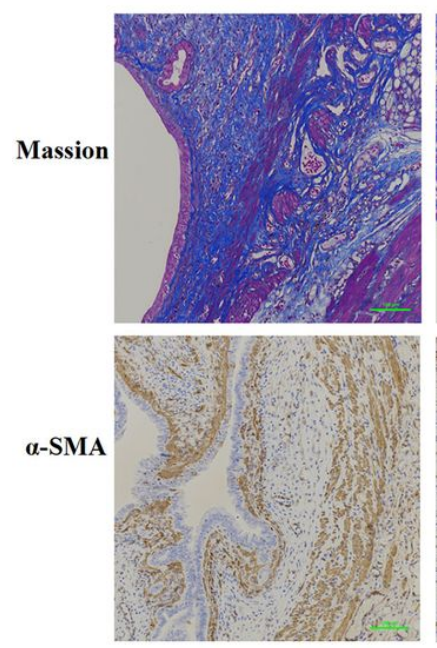

Model_Ecto
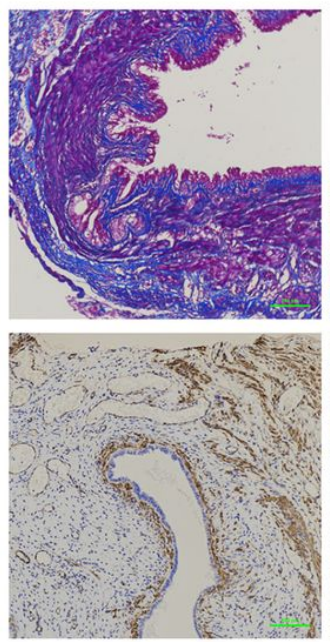

ELC High_Ecto
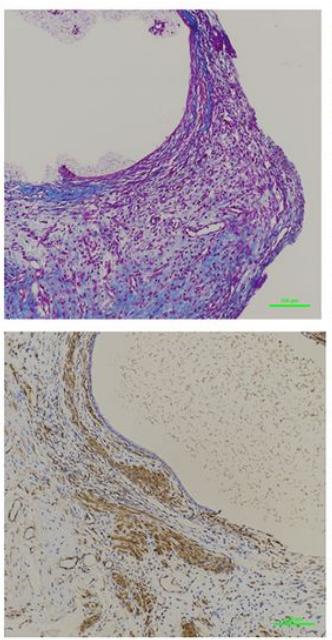

ELC Middle_Ecto
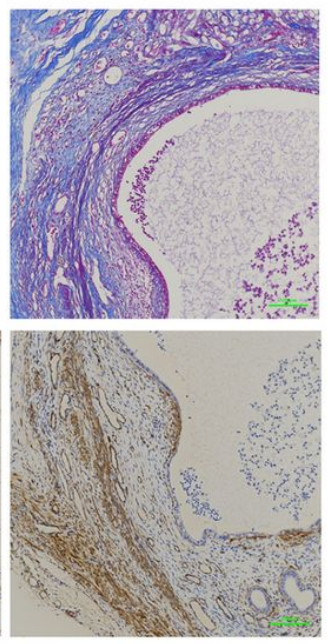

ELC Low_Ecto

Massion trichrome staining

a-SMA
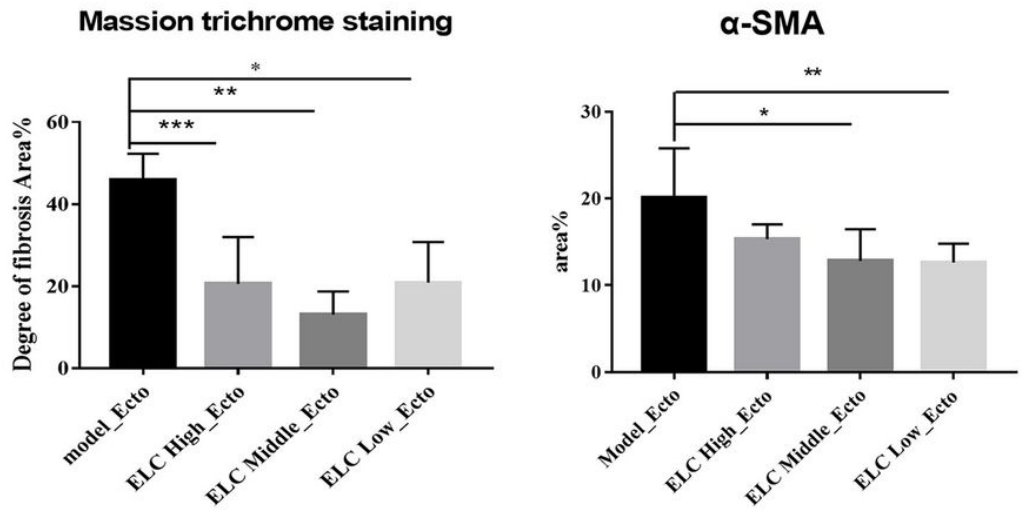

\section{Figure 6}

The result of fibrosis in ectopic lesion in endometriosis model rats Values are represented as mean $\pm S D, n=4$. $p<0.05$ as determined by one-way ANOVA analysis. The Massion showed local fibrosis after modeling $(\times 200)$. The percentage of fibrosis was positive by massion staining of ectopic lesions in tissue sections. Compared with the model group, the ELC group has a lower degree of ELC fibrosis than the model group, as mean $\pm S D(n=4)(* P<0.05)$. ELC could reduce the degree of fibrosis of the lesion.Model group:45.86 $\pm 6.42 \%$,ELC high_Ecto:20.56 $\pm 11.41 \%$,ELC Middle_Ecto:13.06 $\pm 5.680 \%, E L C$ low_Ecto:20.869 $\pm 9.9294 .{ }^{*} \mathrm{P}=0.0068,{ }^{* *} \mathrm{P}=0.0009,{ }^{* * *} \mathrm{P}=0.0074(\mathrm{P}<0.05)$. The expression of a-SMA.Compared with the control eutopic endometrium, the fibrosis area (Area\%) of the model group and ELC group were increased significantly $(P=0.0457<0.05)$. Compared with the model group, the fibrosis area ratio was reduced in ELC middle-dose group and low-dose group. *P=0.040, ** $\mathrm{P}=0.0346(\mathrm{P}<0.05)$. 
A
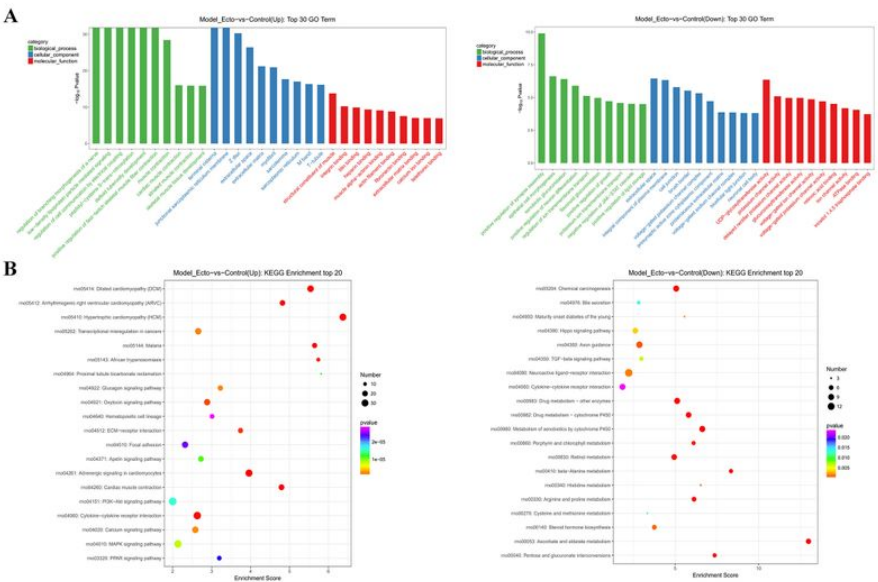

C
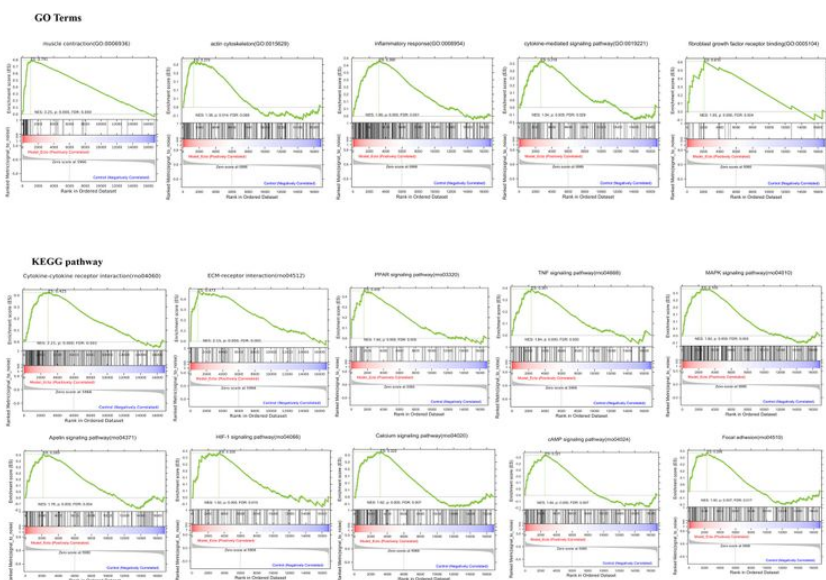

\section{Figure 7}

The result of gene expression profifiling of the rat endometriosis model. RNA-sequencing reveals transcriptome profile of gene expression changes in endometriosis rat models induced by autotransplantation. (A) Results of Gene Ontology enrichment analysis for up-DEGs and down-DEGs reversed by ELC ectopic endometrium groups and model ectopic endometrium groups. (B) KEGG analysis of up and down genes.KEGG pathway analysis of up-regulated and down-regulated genes in ectopic endoemtrium in rats model vs. Control eutopic endometrium. Gene ratio refers to the ratio of the number of target genes associated with a KEGG pathway to the total number of genes in the pathway. (C) Gene Set Enrichment Analysis (GSEA) in endometriosis rats model shows enrichment of GO analysis and KEGG pathway. Normalized enrichment scores (NES), p-value and false discovery rate (FDR) are indicated for each gene set. GSEA revealed a significant enrichment of gene signatures associated with endometriosis $(P<0.05)$. 
A
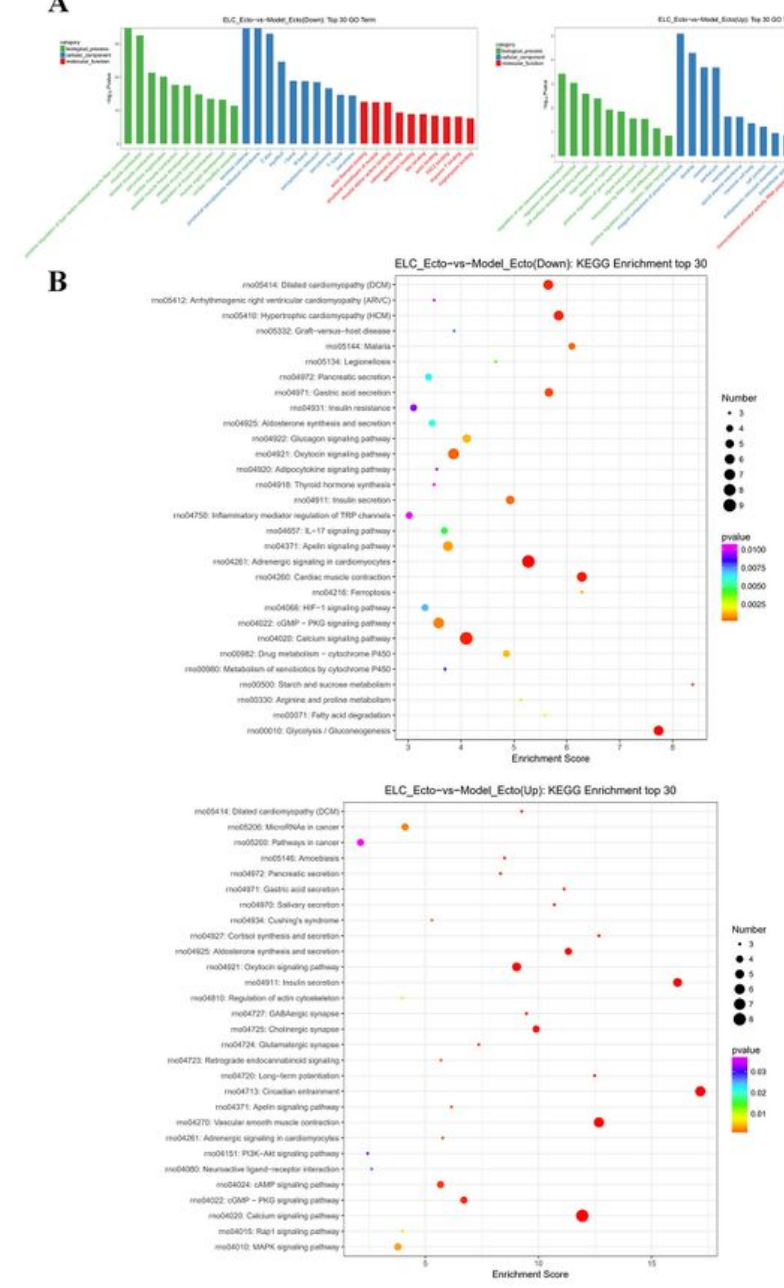

C
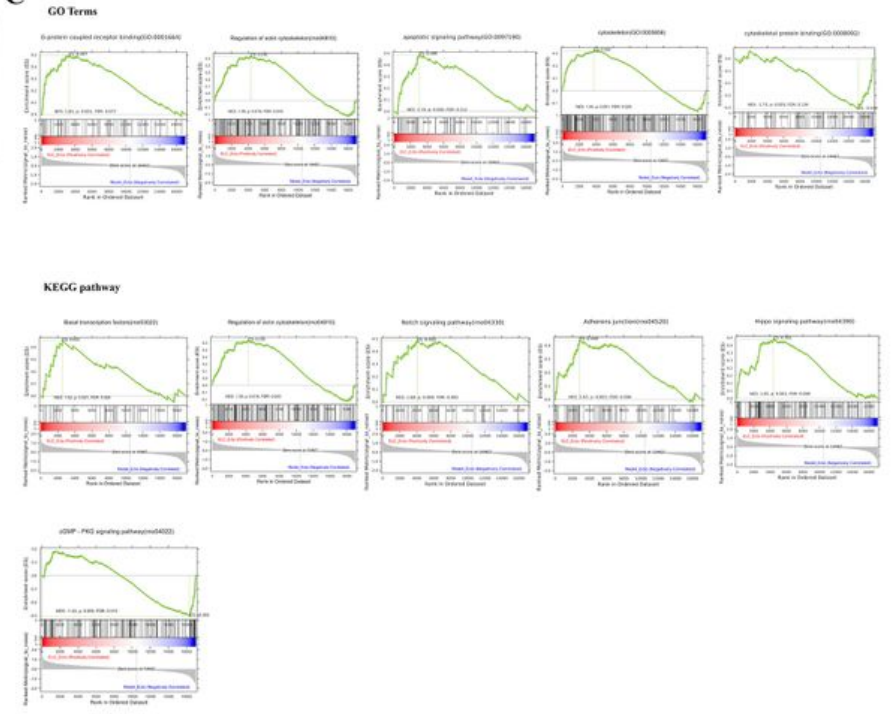

D
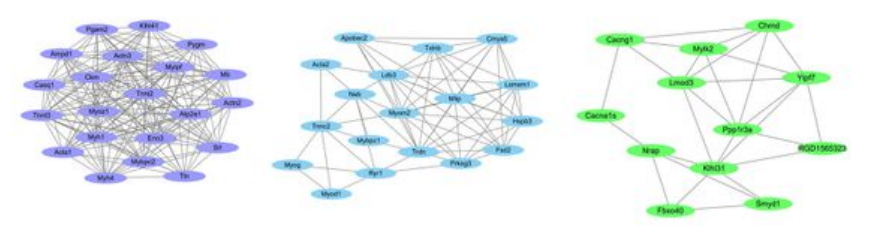

\section{Figure 8}

RNA-sequencing reveals transcriptome profile of gene expression changes in endometriosis rat models and the treatment of ELC capsules of endometriosis rats. (A) Results of Gene Ontology enrichment analysis for up-DEGs and down-DEGs reversed by ELC ectopic endometrium groups and model ectopic endometrium groups. (B) Results of KEGG enrichment analysis between ELC_Ecto groups and Model_Ecto groups. (C) The results of GSEA analysis revealed a significant enrichment of gene signatures associated with ELC treatment $(P<0.05)$. (D) The MCODE network of cluster analysis of the potential targets network in ELC treatment. 
A

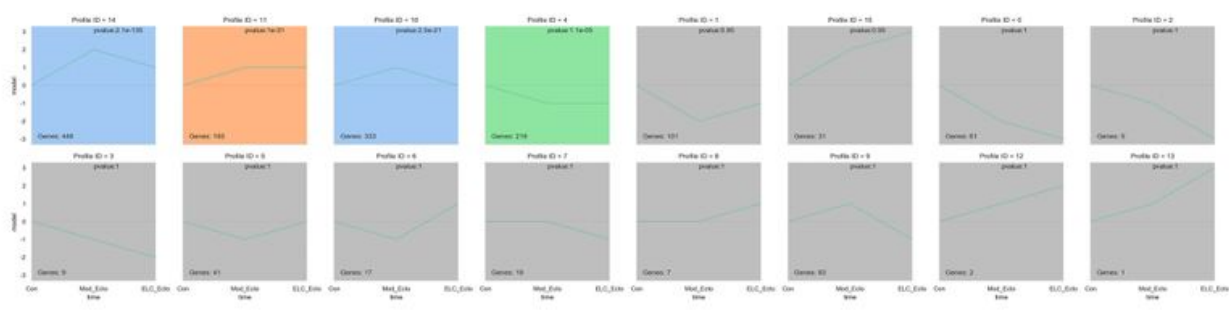

B

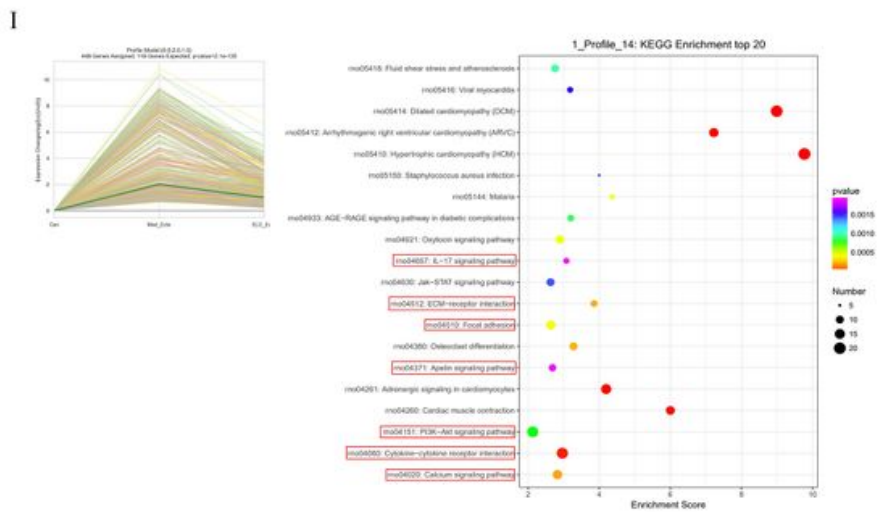

III
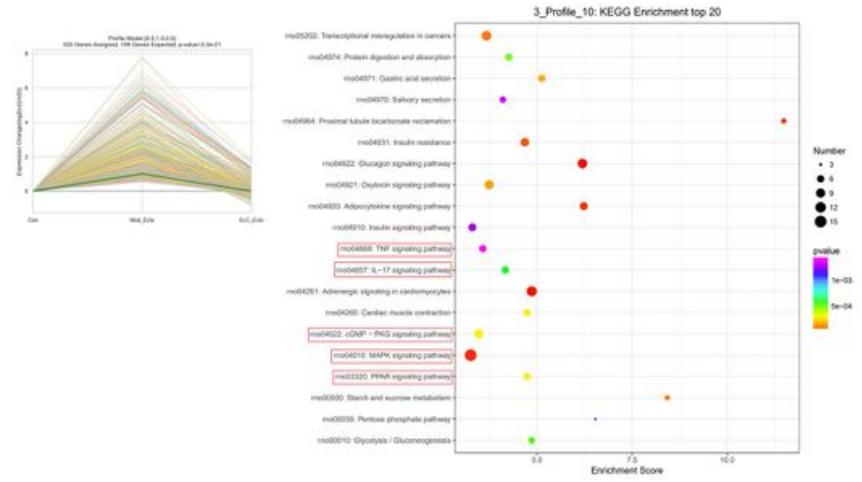

II
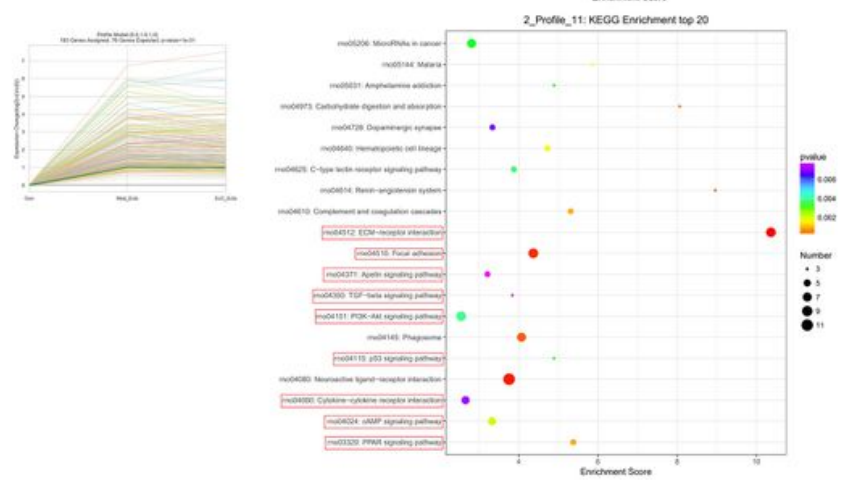

IV
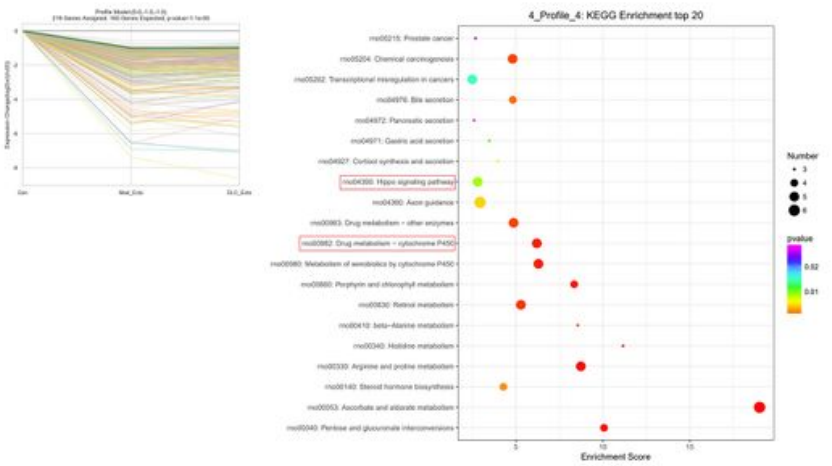

Figure 9

The STEM analysis of ELC treatment in endometroisis rats. (A)The overall STEM analysis. (B)The stem analysis results suggest that there are 4 statistically significant trends. The results of gene cluster analysis were statistically significant in profile of $14,11,10$ and $4(P<0.05)$. The profile_11 (Figure 9 B( $(\mathbb{Z})$ ) and

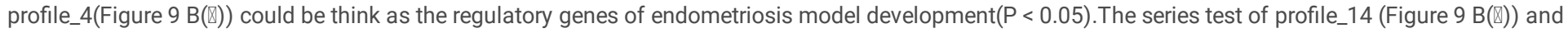
profile_10 (Figure $9 \mathrm{~B}(\mathbb{\nabla})$ ) showed that the significant clusters were considered as potential profiles that could be affected by ELC treatment $(\mathrm{P}<0.05)$. The KEGG pathways of the STEM enrichment genes in profile 14,profile 11,profile 10,and profile 4. 
A

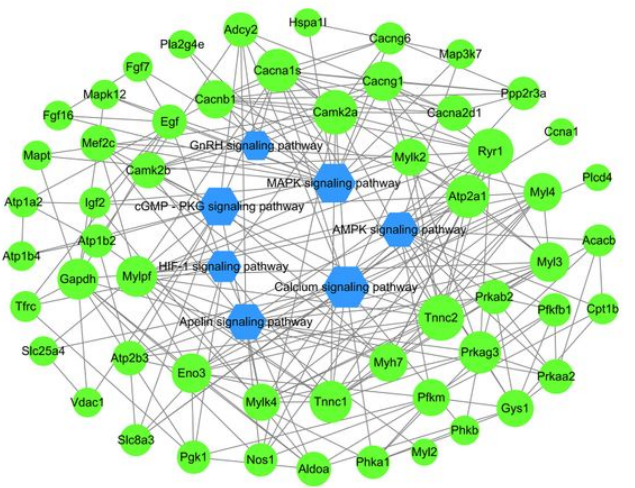

B
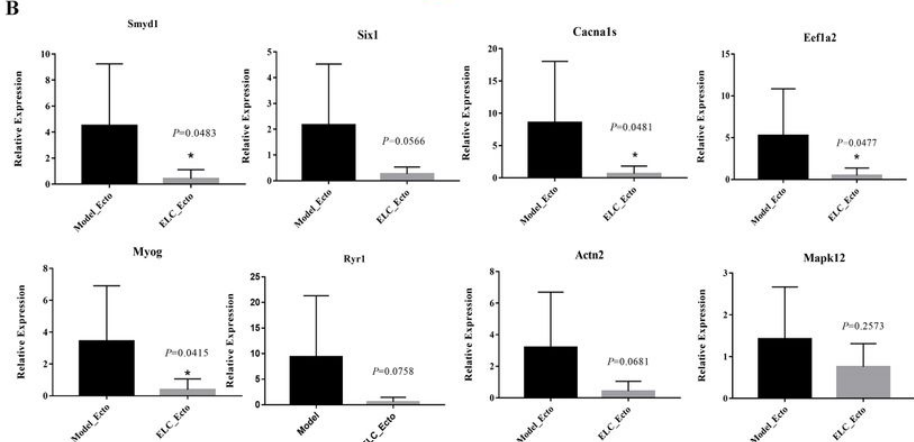

Ryri
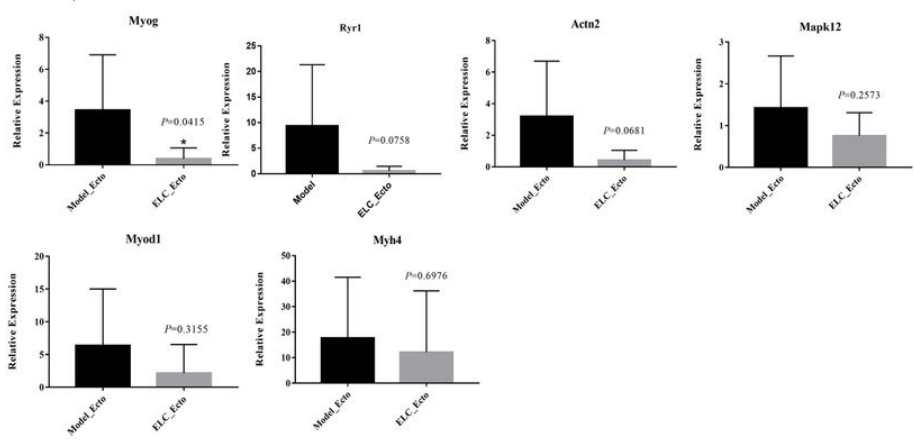

\section{Figure 10}

Effect of ELeng Capsules on gene expression levels of DEGs in the endometriosis model rats. (A) The network of KEGG pathways and major targets in Model_Ecto vs ELC_Ecto down-regulated DEGs. (B) Verification of DEGs by qRT-PCR. Expression of genes in ectopic endometrium tissues in endometriosis rats models were detected by qRT-PCR, and shown by the expression fold changes.ACTB was used as the internal control. Data are shown as means \pm SD, * $P$ $<0.05$.

\section{Supplementary Files}

This is a list of supplementary files associated with this preprint. Click to download.

- 1CompoudsandtargetsofELengCapsules.xIsx

- 2Theresultofmoleculardocking.xls

- 3.Supplementarymaterial3.docx

- 4SupplementaryMaterial4.docx

- 5ThePCAandHeatmapofRNAsequence.tif

- 6TheexpressionofDEGsofModelectovsELCectogroup.docx

- 7SRAofRNAsequence.xlsx 